ARTICLE

DOI: $10.1038 / s 41467-018-07492-4$

\title{
Single-cell RNA sequencing unveils an IL-10- producing helper subset that sustains humoral immunity during persistent infection
}

Gang Xin ${ }^{1}$, Ryan Zander ${ }^{1}$, David M. Schauder ${ }^{2}$, Yao Chen $^{2}$, Jason S. Weinstein ${ }^{3}$, William R. Drobyski ${ }^{2}$, Vera Tarakanova ${ }^{2}$, Joseph Craft $\mathbb{B}^{3}$ \& Weiguo Cui (i) ${ }^{1,2}$

During chronic viral infection, the inflammatory function of CD4 T-cells becomes gradually attenuated. Concurrently, Th1 cells progressively acquire the capacity to secrete the cytokine IL-10, a potent suppressor of antiviral $T$ cell responses. To determine the transcriptional changes that underlie this adaption process, we applied a single-cell RNA-sequencing approach and assessed the heterogeneity of IL-10-expressing CD4 T-cells during chronic infection. Here we show an IL-10-producing population with a robust Tfh-signature. Using IL10 and IL-21 double-reporter mice, we further demonstrate that $\mathrm{IL}-10^{+} \mathrm{IL}-21^{+}$co-producing Th cells arise predominantly during chronic but not acute LCMV infection. Importantly, depletion of IL-10+IL-21+ co-producing CD4 T-cells or deletion of 1170 specifically in Tfh cells results in impaired humoral immunity and viral control. Mechanistically, B cell-intrinsic IL10 signaling is required for sustaining germinal center reactions. Thus, our findings elucidate a critical role for Tfh-derived IL-10 in promoting humoral immunity during persistent viral infection.

\footnotetext{
${ }^{1}$ Blood Research Institute, BloodCenter of Wisconsin, Milwaukee, WI 53213, USA. ${ }^{2}$ Department of Microbiology and Molecular Genetics, Medical College of Wisconsin, Milwaukee, WI 53226, USA. ${ }^{3}$ Department of Immunobiology, Yale University School of Medicine, New Haven, CT 06520, USA. These authors contributed equally: Gang Xin, Ryan Zander, David M. Schauder. Correspondence and requests for materials should be addressed to

R.Z. (email: ryan.zander@bcw.edu) or to W.C. (email: weiguo.cui@bcw.edu)
} 
C D4 T cells display immense versatility in changing their differentiation pattern in the face of persistent lymphocytic choriomeningitis virus (LCMV) infection ${ }^{1}$. Similar to CD8 T cells, CD4 T cells rapidly lose their capacity to produce the effector cytokines IL-2, TNF- $\alpha$, and IFN- $\gamma$ during chronic infection $^{2,3}$. However, CD4 T cells also gradually acquire the capacity to express IL-21 and IL-10 ${ }^{4,5}$, suggesting that continuous antigenic exposure may drive functional adaption within the $\mathrm{T}$ helper cell compartment. Notably the inhibitory role of IL-10 in suppressing T-cell responses during chronic viral infection is welldocumented $^{6-8}$. However, IL-10 signaling may also protect the host against collateral damage caused by excessive and prolonged inflammation ${ }^{9}$. Intriguingly, two recent studies have identified that the regulatory effects of IL-10 may be multifaceted, and can largely depend on the cellular source of IL-10, the responding cell type, and the nature of the infection ${ }^{4,10}$. Although multiple distinct CD4 T-cell subsets, including Tregs, Tr1 cells, and Th1 cells can produce IL-10 in response to viral infection ${ }^{4,10,11}$, the biological consequences of IL-10 derived from T helper cell subsets other than that of Th1 cells remains incompletely understood in the context of persistent infection.

In contrast to the suppressive nature of IL-10, CD4 T-cellderived IL-21 is critical to sustain the function of CD8 T-cells and mediate viral containment during persistent infection ${ }^{5,12-14}$. IL21 is also a potent facilitator of B cell help ${ }^{15}$. Recent evidence suggests that CD4 $\mathrm{T}$ follicular helper (Tfh) cells are the major producers of IL-21 during chronic viral infection ${ }^{1}$. Several studies over the last decade have identified that Tfh cells play a central role in orchestrating the germinal center (GC) reaction, a process that is essential for the selection of high-affinity B cell receptors and the development of long-lived plasma cells and memory $B$ cells ${ }^{16-20}$. Despite the pivotal role of Tfh cells in mediating humoral immunity during chronic infections, the cellular and molecular factors important for Tfh differentiation and function are still being unraveled.

Tfh cells can be distinguished from other CD4 T-cell lineages based on their combinatorial expression of the chemokine receptor CXCR5, the co-stimulatory receptor ICOS, and the transcriptional repressor B cell lymphoma 6 (Bcl-6), all of which are required for Tfh differentiation ${ }^{21,22}$. Additionally, CD4 T-cell expression of SLAM-associated protein (SAP) is essential for facilitating the formation of stable T-cell-B-cell conjugates and is critical for GC Tfh development ${ }^{16,23,24}$. Although the importance of Tfh-secreted IL-21 in maintaining the GC reaction is wellappreciated, several recent reports have identified that Tfh cells display vast heterogeneity in the effector molecules they produce ${ }^{25-29}$. However, the importance of Tfh-derived cytokines other than IL-21 remains less well-defined. In this study, we performed single-cell RNA sequencing (scRNA-seq) to determine the heterogeneity among IL-10-secreting CD4 T cells during persistent viral infection. Unexpectedly, single-cell transcriptomics uncovered a subset of IL-10-producing CD4 T cells with a robust $\mathrm{Tfh}$ signature. Herein, we report that a unique subset of IL- $10^{+} \mathrm{IL}-21^{+} \mathrm{Tfh}$ cells predominantly arise during chronic, but not acute LCMV infection. Importantly, depletion of IL- $10^{+}$IL- $21^{+}$co-producing CD4 T cells or Tfh-specific deletion of IL-10 results in significantly reduced GC reactions, antibody production, and viral control. Collectively, this study highlights the importance of Tfh cells remaining plastic in their ability to produce cytokines so that they can optimally regulate humoral immune responses to establish control over viral replication.

\section{Results}

scRNA-seq reveals a subset of $\mathbf{I L}-10^{+}$Tfh cells. To investigate the transcriptional heterogeneity of IL-10-producing CD4 T cells responding to chronic viral infection, we performed scRNA-seq on IL-10-expressing CD4 T cells from mice infected with persistent LCMV Cl13. To do this, 10BiT reporter mice (which possess a BAC transgene containing the Illo gene locus with the Thy1.1 cDNA insertion ${ }^{30}$ ) were infected with LCMV Cl13, and on day 16 post infection (p.i.), we sort-purified Thy1.1+(IL-10 $\left.{ }^{+}\right)$ CD4 $\mathrm{T}$ cells specific for the $\mathrm{I}-\mathrm{A}^{\mathrm{b}}$ restricted $\mathrm{GP}_{61-80}$ epitope of LCMV (Fig. 1a). Next, we used the 10X Genomics Chromium system to generate a scRNA-seq library (Fig. 1a), and a downstream transcriptional analysis was performed on 628 IL-10expressing CD4 T cells.

Notably, IL- $10^{+} \mathrm{CD} 4 \mathrm{~T}$ cells grouped distinctly into eight clusters when visualized by $t$-distributed stochastic neighborembedding analysis ( $t$-SNE) projection (Fig. 1b). As expected, many IL-10-expressing CD4 T cells co-expressed the gene Tbx21, which encodes the Th1-defining transcription factor T-bet (Fig. 1c). Moreover, genes encoding Th1-associated molecules granzyme $\mathrm{B}, \mathrm{Ccl} 3$, Cxcr6, and Id2 were also highly expressed in most of these subpopulations (Fig. 1c, d and Supplementary Figure 1A). In particular, clusters $2-8$, coordinately displayed high expression of these Th1-signature genes (Fig. 1c, d), suggesting that these subpopulations may be of the Th1 lineage. Additionally, the Blimp-1-encoding gene $\operatorname{Prdm1}$, which has recently been identified as being essential for $\mathrm{T}$-cell production of IL-10 4,31 , was ubiquitously expressed and was detected among all eight IL-10 ${ }^{+}$T-cell clusters (Fig. 1c). Similarly, each cluster displayed high expression levels of Maf (encodes c-Maf), a potent inducer of IL-10 expression ${ }^{32,33}$ (Supplementary Figure 1B). Intriguingly, our scRNA-seq analysis also identified a unique cluster of IL-10-expressing CD4 T cells that displayed high expression of Tfh signature genes, including Cxcr5, Il21, Tcf7, and Il6ra (Fig. 1c, d). Moreover, Bcl6 expression was also enriched within this Tfh-like subset (Fig. 1c). Of note, all eight clusters of IL- $10^{+} \mathrm{CD} 4 \mathrm{~T}$ cells displayed minimal expression of Foxp3 (Supplementary Figure 1C), suggesting the absence of the Treg lineage among $\mathrm{GP}_{61-80}$-specific IL-10-producing CD4 $\mathrm{T}$ cells. Lastly, among the Th1-like clusters 2-8, clusters 7 and 8 displayed relatively higher expression levels of Gzmk, Klf2, Lgals1, and Lgals3, indicating that these two subgroups may be at a different stage of differentiation compared to their Th1-like effector counterparts (Supplementary Figure 1D). Collectively, our scRNA-seq analyses highlight that IL-10-producing CD4 T cells responding to chronic viral infection are comprised of multiple transcriptionally distinct subsets.

IL-10 ${ }^{+}$IL-21 ${ }^{+}$Tfh cells form during chronic but not acute LCMV. Our finding that a subset of IL-10-expressing CD4 T cells displays a robust $\mathrm{Tfh}$ signature was highly unexpected, and suggests that Tfh cells may also display remarkable heterogeneity. It also brings to question whether the development of IL- $10^{+} \mathrm{Tfh}$ cells is a distinct feature of chronic viral infections. Thus we next performed comparative studies to determine whether acute or chronic viral infection differentially supports the formation of IL$10^{+} \mathrm{IL}-21^{+}$co-producing Tfh cells. To do this, we crossed 10BiT mice with IL-21-turbo red fluorescent protein (IL-21tRFP) reporter mice ${ }^{12,25,34}$ to generate $10 \mathrm{BiT}-I 121 \mathrm{tRFP}$ double-reporter mice. We then infected separate groups of 10BiT-Il21tRFP mice with LCMV Armstrong (Arm) or LCMV Cl13 to establish an acute or chronic viral infection, respectively. Of note, and in align with previous reports ${ }^{4,25,34}$, we found that Thy1.1 and IL-21-tRFP expression closely correlated with IL-10 and IL-21 protein production, respectively in virus-specific CD4 T cells (Supplementary Figure $2 \mathrm{~A}, \mathrm{~B})$. On day 10 p.i., the proportion of $\mathrm{CXCR} 5^{+} \mathrm{PD}$ -

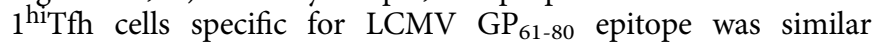
between LCMV-Arm and LCMV Cl13-infected mice (Fig. 2a). 
a

Infect 10Bit reporter mice with LCMV Cl13

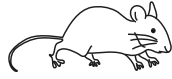

16 days

Sort Thy1.1 (IL-10+)

$\mathrm{CD}^{+}$CD44 $4^{\text {hi }}$ GP66:I-A

-specific splenic CD4 T cells

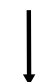

Perform single cell RNA sequencing using $10 \mathrm{X}$ genomics b

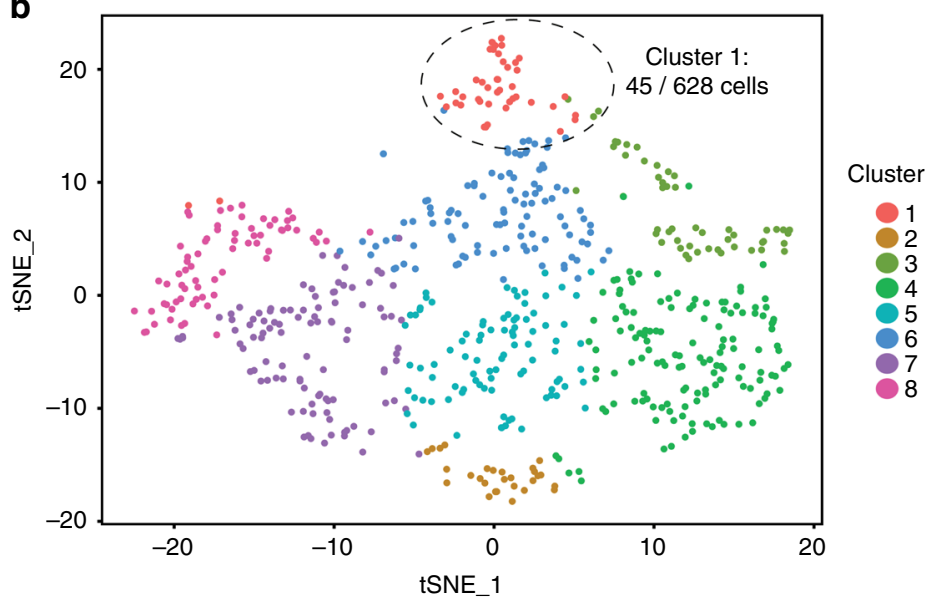

C

Th1 signature genes

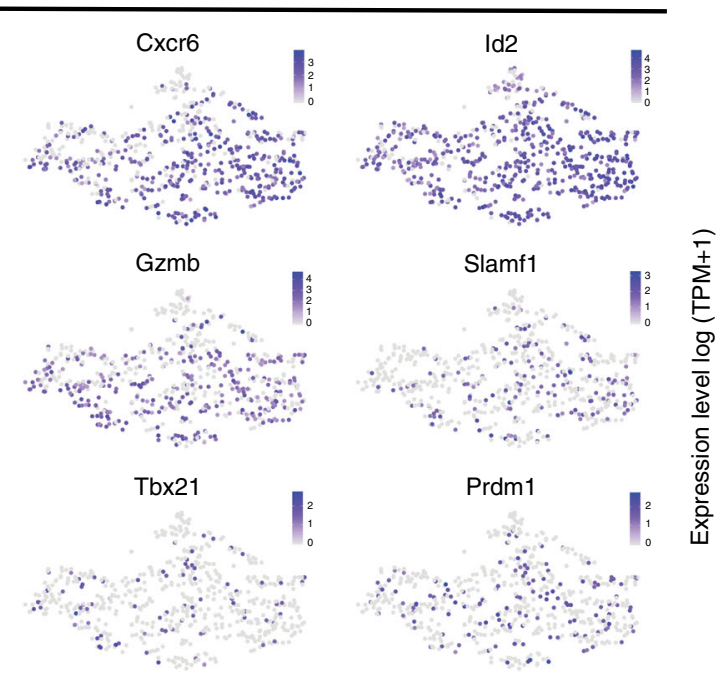

Tfh signature genes
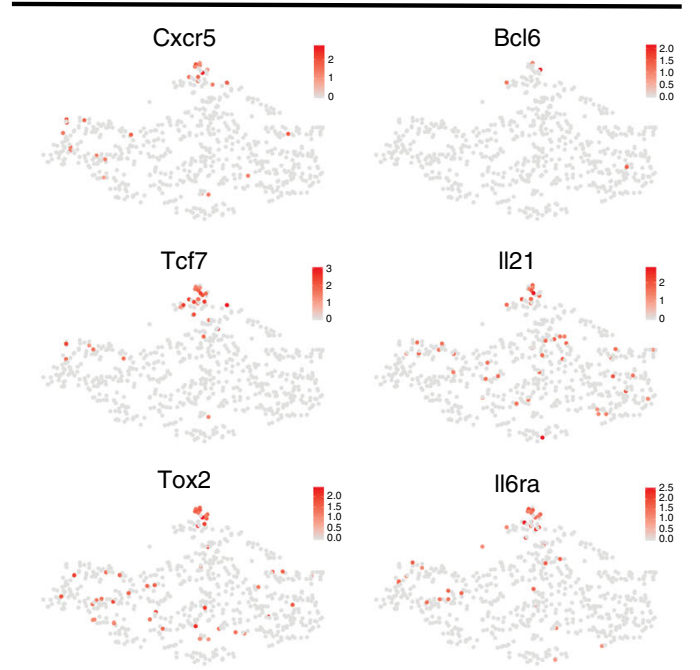

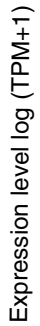

d
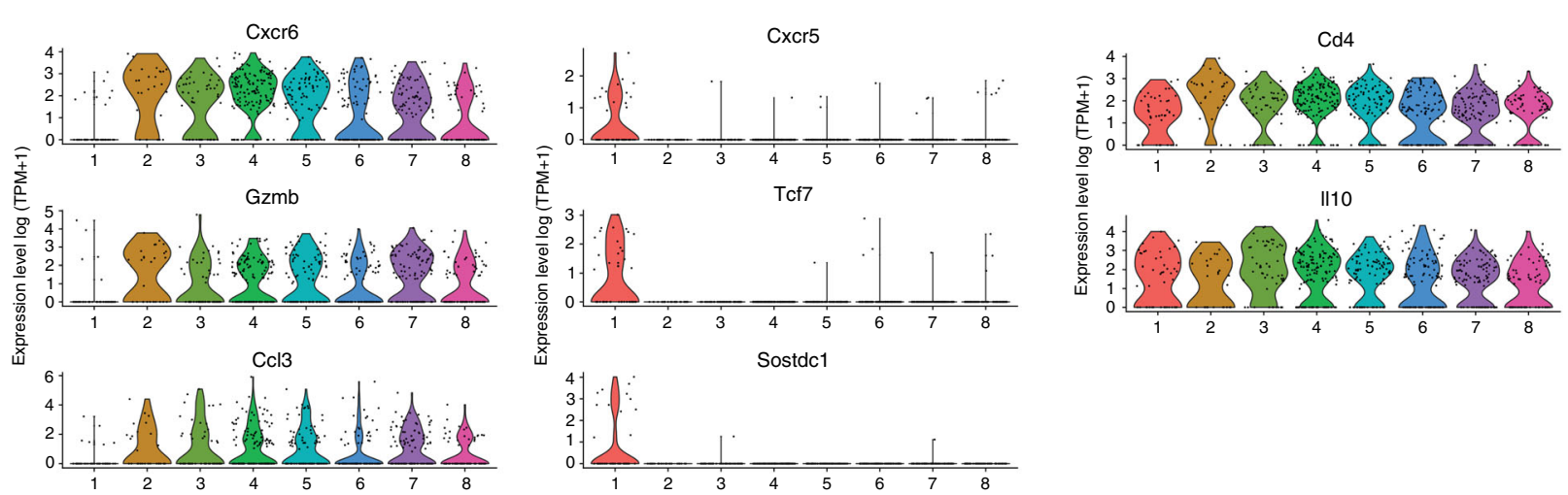

Fig. 1 ScRNA-seq of LCMV-specific IL-10+CD4 T cells. a Outline of experiment. b t-SNE projection of 628 IL-10-producing CD4 T cells from LCMV CI13infected mice at day 16 p.i. colored by cluster. c $t$-SNE plots showing relative expression of Th1 (left) and Tfh (right) signature genes in each cell. $\mathbf{d}$ Violin plots depicting expression of control genes (Cd4, I110), Th1 genes (Cc/3, Cxcr6, Gzmb), and Tfh genes (Cxcr5, Tcf7, Sostdc1). See also Figure S1

Consistent with prior studies ${ }^{1,35}$, splenic Tfh cells from either acute or chronic LCMV-infected mice were highly competent to

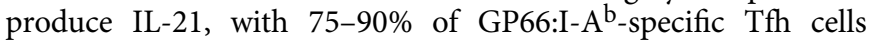
actively producing IL-21 directly ex vivo (Fig. 2a). Strikingly, whereas Tfh cells responding to acute LCMV-Arm displayed a negligible capacity to co-produce both IL-10 and IL-21, $10-15 \%$ of virus-specific and total effector Tfh cells from LCMV Cl13infected mice were competent to co-produce IL-10 and IL-21 (Fig. 2a). This pattern of IL- $10^{+} \mathrm{IL}-21^{+}$dual-producing Tfh cells emerging primarily during chronic LCMV infection was temporally sustained (Fig. 2b, Supplementary Figure 2C-E).

To determine whether a more prolonged acute viral infection can result in the induction of IL- $10^{+} \mathrm{IL}-21^{+}$Tfh cells, we infected 10BiT-Il21tRFP reporter mice with influenza A/PR8/34 (PR8), which is eliminated from the host within 10 days. Intriguingly, a minor population of Tfh cells responding to PR8 infection also co-produced IL-10 and IL-21 (Supplementary Figure 2F), suggesting that certain acute infections may also spur the 
a
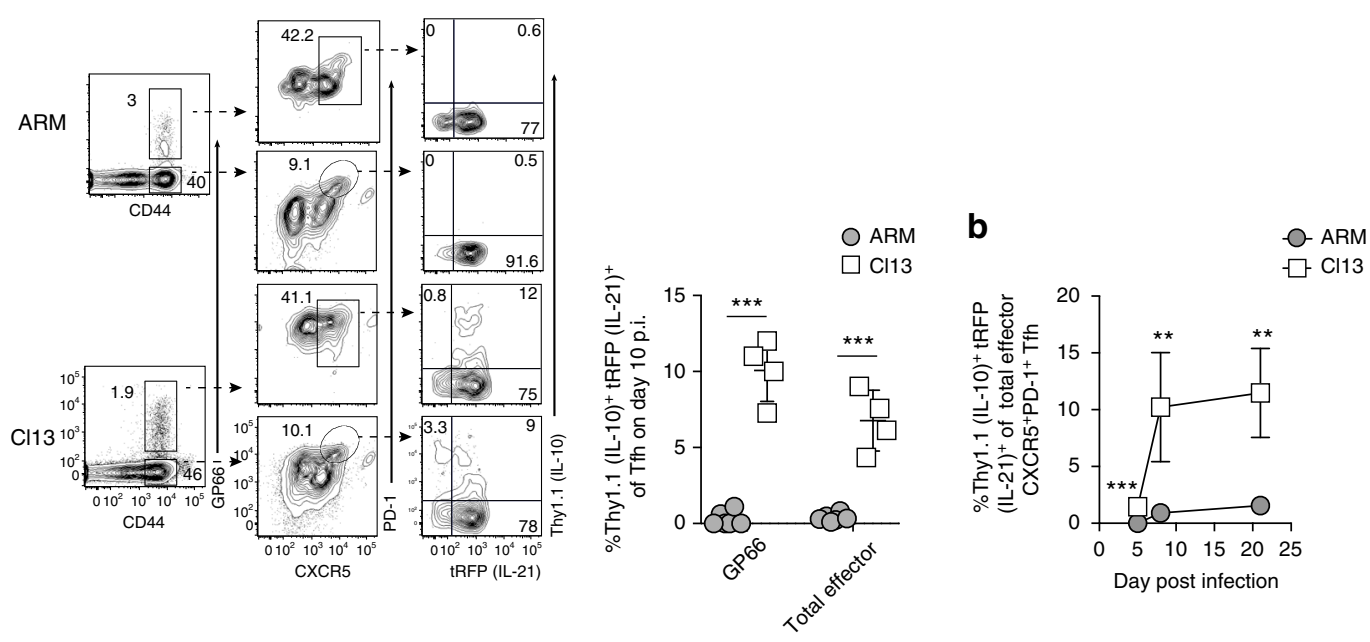

C
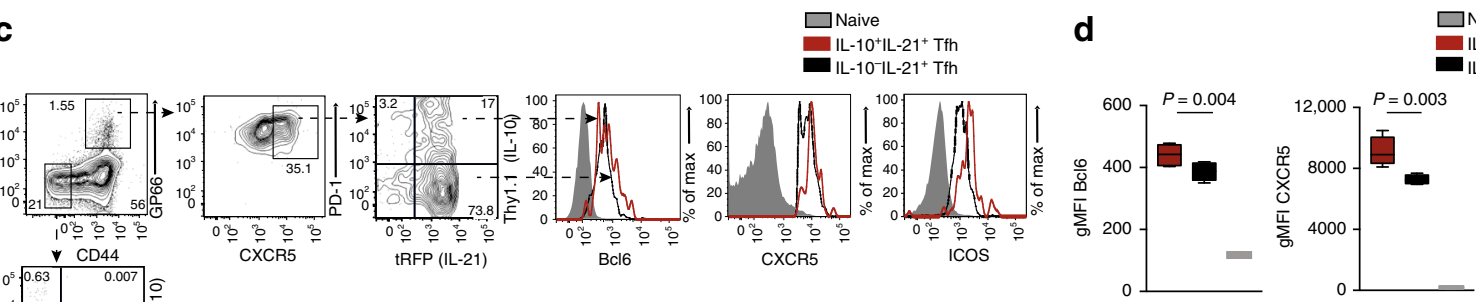

Naive

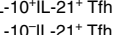

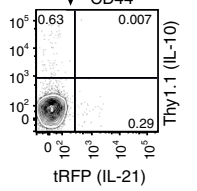

g
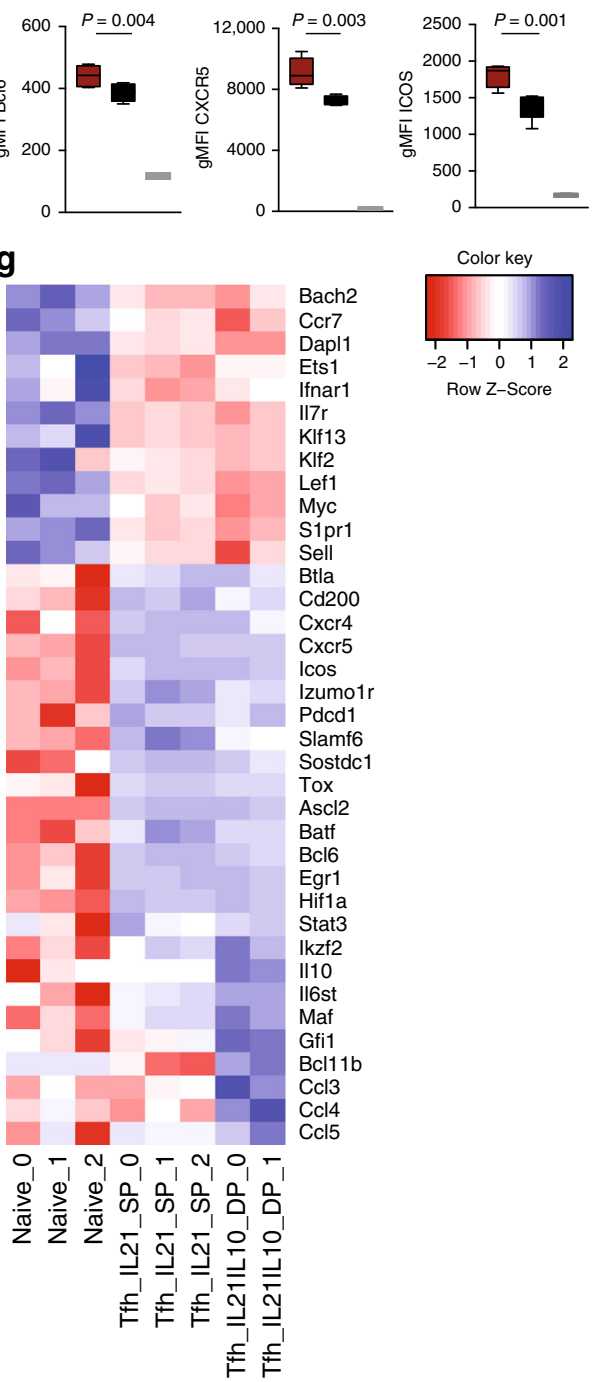

e

$\square$ CD4 $\square \operatorname{lgD} \square$ Thy1.1 (IL-10) $\square$ IL-21-tRFP $\square$ Thy1.1 (IL-10 ${ }^{+}$IL-21-tRFP+ CD4 $^{+}$
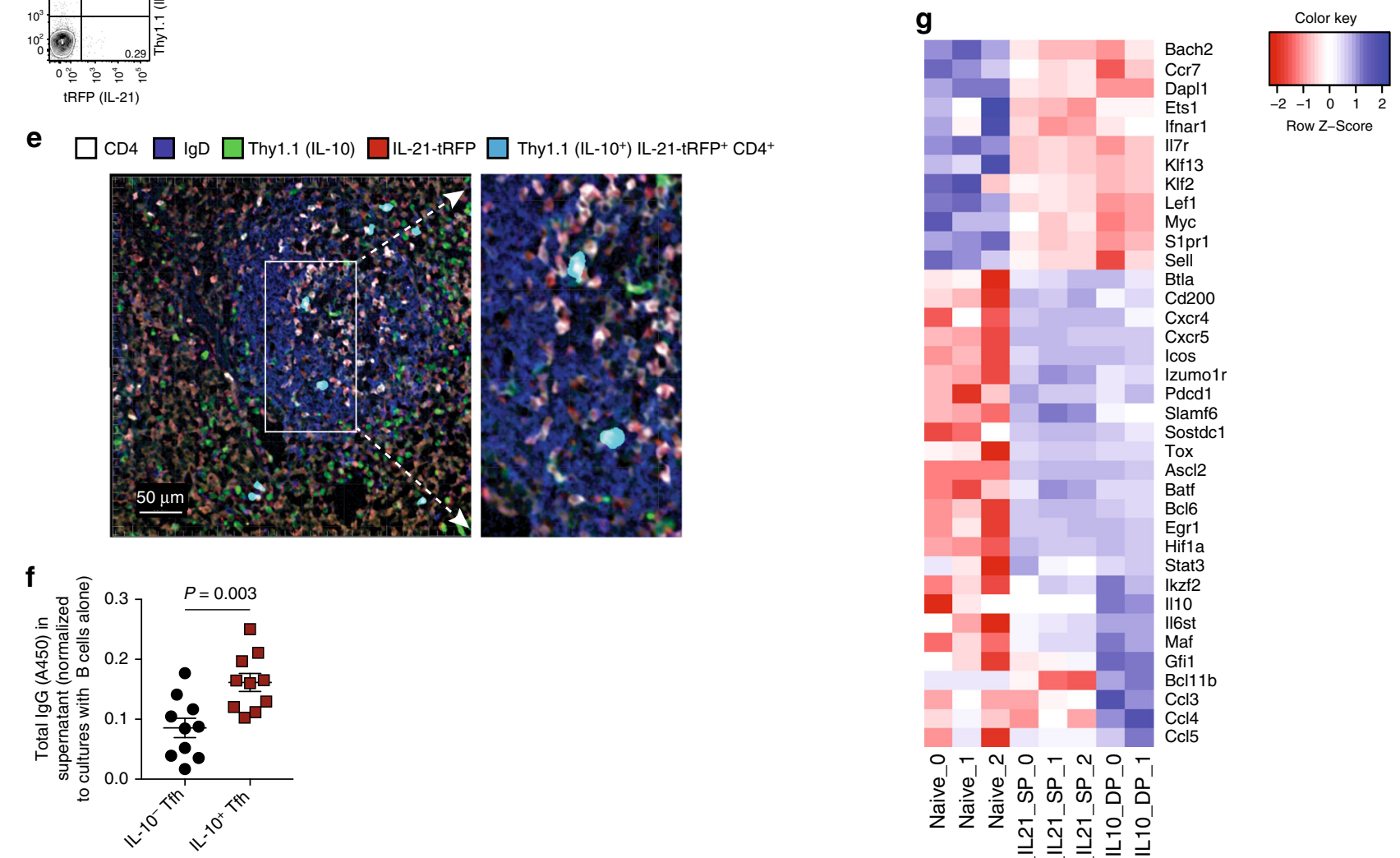

We next investigated whether additional phenotypic and

development of $\mathrm{IL}-10^{+} \mathrm{IL}-21^{+} \mathrm{Tfh}$ cells. However, in contrast to chronic LCMV infection, IL- $10^{+}$IL- $21^{+}$Tfh cells rapidly declined in influenza-infected hosts (Supplementary Figure 2F). Taken together, these results demonstrate that Tfh cells responding to chronic viral infection exhibit an enhanced and sustained capacity to coordinately produce both IL-10 and IL-21. functional differences exist between IL- $10^{+} \mathrm{IL}-21^{+}$and IL-10-IL$21^{+}$Tfh cells during LCMV Cl13 infection. An assessment of Tfhassociated molecules revealed that the relative expression of Bcl-6 was increased by $\sim 25 \%$ in IL- $10^{+} \mathrm{IL}-21^{+} \mathrm{GP} 66^{+} \mathrm{Tfh}$ cells compared with IL-10- $\mathrm{IL}-21^{+}$Tfh cells (Fig. $2 \mathrm{c}$, d). As Bcl-6 is critical for 
Fig. 2 IL-10+ IL-21+ Tfh cells largely develop during chronic but not acute LCMV. a, b 10BiT-II21-RFP mice were infected with either LCMV Arm or LCMV CI13, and splenic $\mathrm{CD} 44^{+}$CD4 T cells were examined on day 10 p.i. a Representative flow plots and summary data displaying the frequency of CXCR5 ${ }^{+}$PD-1 ${ }^{\text {hi }}$ effector and GP66:I-A $\mathrm{A}^{\mathrm{b}}$-specific Tfh cells and subsequent expression of IL-10(Thy1.1) and IL-21(tRFP). b Summary kinetics showing the proportion of IL-10+IL$21^{+}$Tfh cells during LCMV CI13 and ARM infection. c-g 10BiT-II21-RFP mice were infected with LCMV CI13. c, d Flow plots (c) and summary data (d) depicting the relative expression of $\mathrm{BCl}-6, \mathrm{CXCR} 5$ and ICOS on IL-10+ IL-21+ and IL-10-IL-21+GP-66+Tfh cells on day 14 p.i. Gray histograms show the relative expression of these molecules in naive (CD44 ${ }^{\text {o }}$ ) CD4 T cells. e Microscopy of GCs from 10BiT-II21-RFP mice on day 21 p.i.; stained for lgD (blue) CD4 (white) Thy1.1 (green), and IL-21(red). White arrowheads point to Thy1.1+ (IL-10)IL-21+CD4 T cells found in B cell follicle. f Thy1.1+(IL-10) ${ }^{+}$and Thy1.1-(IL-10-) Tfh cells were sort-purified from LCMV Cl13-infected 10BiT-II21-RFP mice on day 14 p.i. and co-cultured with Naive (IgM+ B220 ${ }^{+}$) B cells in the presence of $\alpha$ ntiCD3 and $\alpha$ ti-lgM antibodies as previously described ${ }^{36}$. Summary graph (f) showing the relative amount of total lgG secreted in the supernatant after 5 days of culture. $\mathbf{g}$ Heatmap generated from bulk RNA-sequencing analysis depicting relative expression of select genes in IL- $21^{+}$and IL-10+ IL-21+Tfh cells as compared to naïve CD4 T cells. IL-21+ and IL-10+IL-21+ Tfh cells (CD44 ${ }^{\text {hi }}$ CXCR5+ PD- ${ }^{\text {hi }}$ ) were sorted from the spleens of LCMV Cl13-infected mice on day 18 p.i. FPKM values were log-transformed and then centered and scaled for each row. Summary data (mean $+/-$ SD in $\mathbf{a}$ and $\mathbf{b}$, or box and whiskers plot showing median (midline) and interquartile range with upper and lower whiskers representing the range of data distribution in d) are from 4 to 6 mice/group and are representative of $2-3$ independent experiments. Summary data (mean $+/-$ SEM in $\mathbf{f}$ ) are pooled data from three independent experiments. ${ }^{\star} P<0.05$, ${ }^{\star \star} P<0.01,{ }^{\star \star \star} P<0.0001,($ N.S. $=$ not significant). Data were analyzed using two-tailed unpaired student's $t$ tests. See also Figure S2

coordinating Tfh differentiation and function ${ }^{16}$, these data suggest that $\mathrm{IL}-10^{+} \mathrm{IL}-21^{+} \mathrm{Tfh}$ cells may be more GC-like in nature as compared to their IL-10-IL- $21^{+}$Tfh counterparts. In alignment with this idea, GP66:I-A ${ }^{\mathrm{b}}$-specific IL-10+ ${ }^{+} \mathrm{IL}-21^{+}$Tfh cells also displayed $25-40 \%$ increases in their relative expression of ICOS and CXCR5 (Fig. 2c, d), indicating that IL-10 ${ }^{+} \mathrm{IL}-21^{+}$dual-producing Tfh cells may have enhanced trafficking to or retention within the B-cell follicle. Correspondingly, IL- $10^{+} \mathrm{IL}-21^{+} \mathrm{CD} 4 \mathrm{~T}$ cells were found to localize in close proximity to B-cell follicles and could readily be detected within the GC (Fig. 2e and Supplementary Figure 2G). Lastly, we identified that naive $\operatorname{IgM}^{+} \mathrm{B} 220^{+} \mathrm{B}$ cells class-switched and secreted higher amounts of IgG (Fig. 2f) when co-cultured with sort-purified IL- $10^{+} \mathrm{Tfh}$ cells as compared with IL-10-Tfh cells, suggesting that Tfh-derived IL-10 may promote B cell-secreted antibody responses in vitro.

To determine whether IL- $10^{+} \mathrm{IL}-21^{+}$Tfh cells are transcriptionally distinct from IL- $21^{+}$single-producing Tfh cells, we sorted these respective populations from LCMV Cl13-infected reporter mice on day 18 p.i. and performed RNA-seq. As expected, Il10 expression levels were significantly elevated in IL$10^{+} \mathrm{IL}-21^{+} \mathrm{Tfh}$ cells as compared with their IL-21-singleproducing counterparts (Fig. 2g). Intriguingly, RNA-seq analyses also indicated that expression of Maf was increased $>$ twofold in IL- $10^{+}$Tfh cells (Fig. $2 \mathrm{~g}$ ). In addition, several genes encoding various transcription factors, chemokines, and cell surface ligands and receptors were differentially expressed between these two subsets (Fig. $2 \mathrm{~g}$ ). Thus, IL-10+ $\mathrm{IL}-21^{+}$Tfh cells and IL- $21^{+}$singleproducing $\mathrm{Tfh}$ cells are transcriptionally distinct $\mathrm{T}$ helper cell populations.

Of note, we did not detect Foxp3 expression at levels above background in either IL-10 ${ }^{+}$or IL-10-GP-66-specific Tfh cells (Supplementary Figure 3A). However, $\sim 10-15 \%$ of total IL- $10^{+}$CXCR5 ${ }^{+}$PD- 1 hiCD 44 hi CD4 $\mathrm{T}$ cells stably expressed Foxp3 during LCMV Cl13 infection (Supplementary Figure 3A, C). A similar but slightly reduced proportion of Foxp3-expressing CXCR5 ${ }^{+} \mathrm{PD}-\mathrm{h}^{\text {hi }} \mathrm{CD} 44^{\text {hi }} \mathrm{CD} 4 \mathrm{~T}$ cells was also observed in LCMV Cl13-infected Foxp3-YFP reporter mice (Supplementary Figure $3 \mathrm{~B}$ ). Additionally, and consistent with previous reports ${ }^{36,37}$, we identified that Foxp $3^{+} \mathrm{CXCR}^{+} \mathrm{PD}-1^{\text {hi }} \mathrm{CD} 4 \mathrm{~T}$ cells displayed increased expression levels of CTLA4, CD25, and GITR, but lower levels of IL-21, as compared with Foxp3-Tfh cells (Supplementary Figure 3C). Taken together, these data suggest that the majority of $\mathrm{IL}-10^{+} \mathrm{IL}-21^{+} \mathrm{CXCR} 5^{+} \mathrm{PD}-1^{\text {hi }} \mathrm{CD} 4 \mathrm{~T}$ cells that form during persistent viral infection are of the Tfh lineage, and are therefore distinct from Foxp3-expressing $\mathrm{T}$ follicular regulatory cells (TFRs).

$\mathrm{IL}^{-10^{+}}{ }^{+} \mathrm{IL}-21^{+} \mathrm{CD} 4 \mathrm{~T}$ cells are essential to sustain GC reaction. Considering the importance of $\mathrm{B}$ cell-secreted antibody responses in controlling persistent infection ${ }^{1}$, we hypothesized that coordinate production of IL-10 and IL- 21 by CD4 T cells may be essential for sustaining virus-specific antibody responses. To test this, we took advantage of Thy1.1 expression on the surface of IL-10-producing cells in the 10BiT model to selectively deplete IL- $10^{+} \mathrm{IL}-21^{+}$co-producing CD4 T cells in mixed bone marrow (MBM) chimera experiments. To do this, we reconstituted lethally irradiated $C d 4^{-/-}$mice with $\mathrm{MBM}$ cells from the following donors: $C d 4^{-/-}(70 \%)+I l 21^{-/-}(15 \%)+10$ BiT-Il21tRFP $(15 \%)$ as shown in Fig. 3a. In doing so, CD4 T cells in MBM chimeric mice will only be derived from $I l 21^{-/-}$and 10BiT-Il21tRFP in a 50:50 ratio, while the rest of the immune system remains largely wild type (WT, $70 \%$ from $C d 4^{-/-}$). This approach allows for selective depletion of IL- $10^{+} \mathrm{IL}-21^{+} \mathrm{CD} 4 \mathrm{~T}$ cells by administering anti-Thy1.1 depletion antibodies, while $50 \%$ of both IL-10 and IL21 single-producing CD4 T cells will remain intact (Fig. 3a). To generate control MBM chimera mice that would contain approximately equivalent numbers of IL-10 and IL-21 singleproducing T-cells (yet retain $\mathrm{IL}-10^{+} \mathrm{IL}-21^{+} \mathrm{T}$-cells), we reconstituted lethally irradiated $C d 4^{-/-}$mice with MBM cells from the following donors: $C d 4^{-/-}(70 \%)+\mathrm{Il} 10^{-/-} \mathrm{Il}_{2} 1^{-/-}(15 \%)+$ 10BiT-Il21tRFP (15\%), as shown in Fig. 3a. These experimental mice were then challenged with LCMV Cl13, and we proceeded to administer anti-Thy1.1 depletion or IgG2a isotype control antibodies to these groups of mice on days 4 and 6 p.i., before conducting analyses on day 21 p.i. The efficacy in depleting Thy $1.1^{+}\left(\mathrm{IL}-10^{+}\right)$Tfh cells was $>90 \%$ (Fig. $3 \mathrm{~b}$ and Supplementary Figure 4A). Of note, we did not observe any significant difference between groups in the total number of effector CD4 $\mathrm{T}$ cells responding to LCMV Cl13 infection, nor in the proportion or total number of $\mathrm{IL}-21^{+}$single-producing Tfh cells (Fig. 3b and Supplementary Figure 4A). However, depletion of IL- $10^{+} \mathrm{IL}-$ $21^{+} \mathrm{CD} 4 \mathrm{~T}$ cells during the first week of $\mathrm{Cl} 13$ infection precluded the development of IL- $10^{+} \mathrm{IL}-21^{+}$Tfh cells by day 21 p.i. (Fig. $3 \mathrm{~b}$ and Supplementary Figure 4A), suggesting that early differentiation signals may be necessary for the induction of this unique Tfh subset. Strikingly, depletion of $\mathrm{IL}-10^{+} \mathrm{IL}-21^{+}$dualproducing CD4 $\mathrm{T}$ cells severely diminished the GC reaction, as indicated by the marked fourfold reduction in the proportion and total number of GL- $7^{+} \mathrm{Fas}^{+} \mathrm{GC}$ B cells (Fig. 3c). Consistent with abrogated GC B cell responses, T-dependent LCMV-specific antibody titers of the IgG1, IgG2a, and IgG2b isotypes were reduced by $\sim 40-60 \%$ in MBM mice depleted of IL- $10^{+}$IL$21^{+} \mathrm{CD} 4 \mathrm{~T}$ cells (Fig. 3d), which was further associated with impaired control over viral replication (Fig. 3f). Of note, sera levels of T-independent antibody isotypes IgM and IgG3 were similar between experimental groups (Supplementary Figure 4B). Importantly, depletion of IL- $10^{+} \mathrm{IL}-21^{+} \mathrm{CD} 4 \mathrm{~T}$ cells also resulted in diminished neutralizing antibody titers (Fig. 3e), and linear 
a
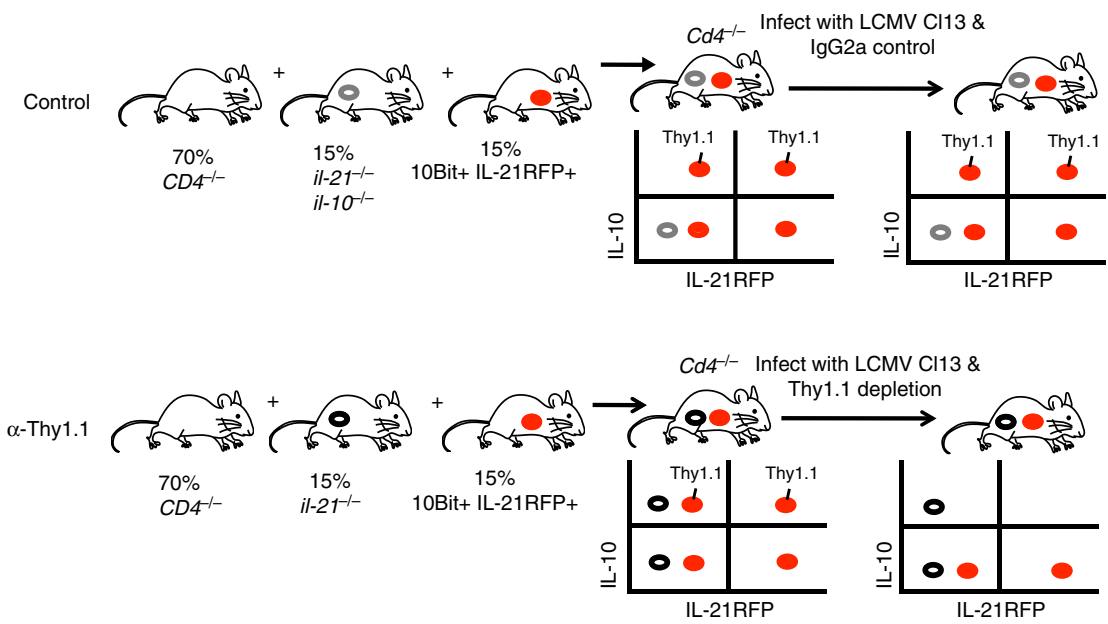

b

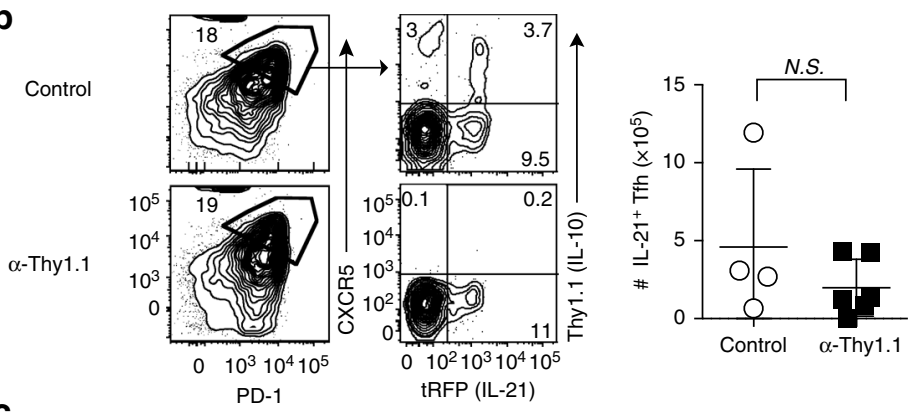

C

PD-1
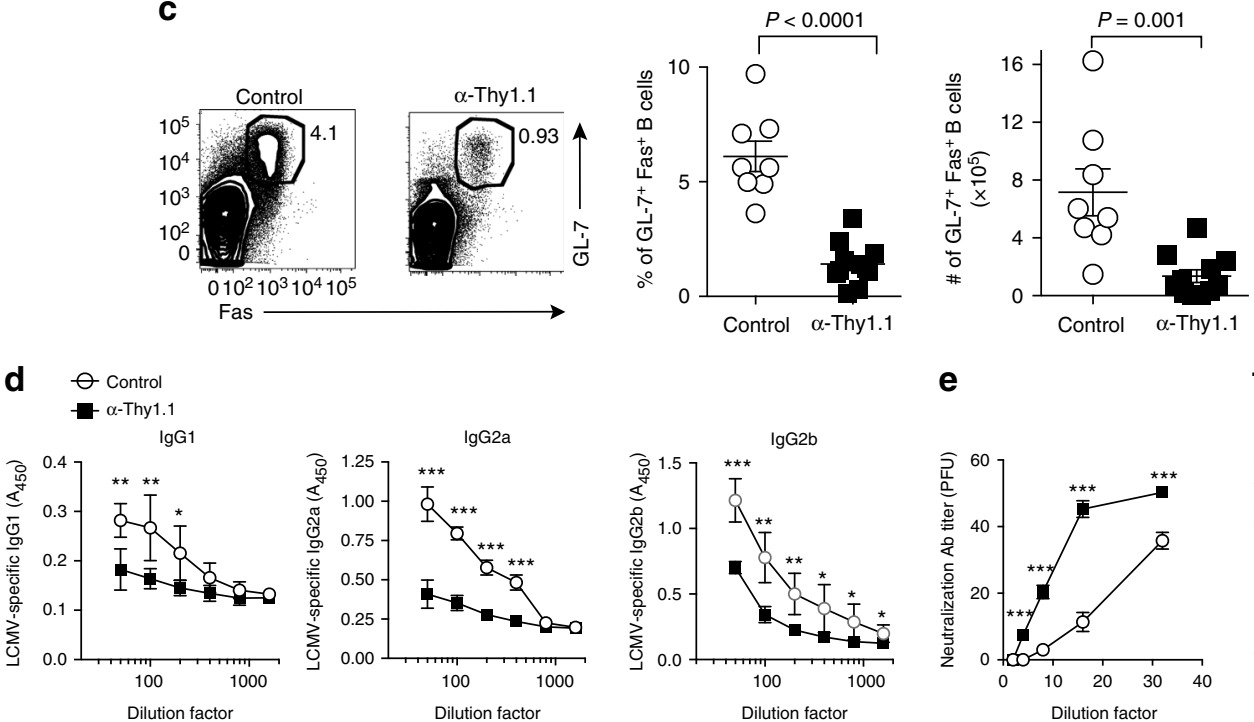

$\mathbf{f}$

Fig. $3 \mathrm{IL}-10^{+} \mathrm{IL}-21^{+} \mathrm{CD} 4 \mathrm{~T}$ cells promote humoral immunity during chronic LCMV. a Experimental scheme showing the generation of MBM chimeric mice and the strategy to delete $\mathrm{IL}-10^{+} \mathrm{IL}-21^{+}$co-producing CD4 T cells. Briefly, CD4 ${ }^{-/}$- mice were lethally irradiated and reconstituted with bone marrow cells from the indicated donors at the ratios indicated. Red dots in the schematic represent bone marrow cells derived from10BiT-II21-RFP donor mice. Gray dots and black dots represent bone marrow derived from il-21-/-IL-10-/- and il-21-/- donor mice respectively. The tables (right) indicate all of the potential IL-10 and IL-21-producing CD4 T cell compartments that bone marrow derived from the indicated donor mice can give rise to. $\mathbf{b}-\mathbf{f} \mathrm{MBM}$ mice were infected with LCMV CI13 and treated with either Thy 1.1 depletion or isotype control antibodies on days 4 and 6 p.i. b, $\mathbf{c}$ Representative flow plots and summary data showing the frequency and total number of II-21+CXCR5+ PD- $1^{\text {hi }}$ Tfh cells (b) and GL-7+Fas ${ }^{+}$GC B cells (c) on day 21 p.i. (d) Summary graphs of LCMVspecific serum IgG1, IgG2a, and IgG2b antibodies. e Summary graph showing relative antibody neutralization titers for control and Thy1.1-depleted mice. $\mathbf{f}$ Viral titers were quantified by plaque assay using serum from experimental mice. Summary data (mean +/- SD in b) or (mean +/- SEM in c-f) are from 4 to 6 mice/group per experiment and are representative of two independent experiments. ${ }^{\star} P<0.05,{ }^{\star \star} P<0.01,{ }^{\star \star \star} P<0.0001,(N . S$. $=$ not significant). Data were analyzed using two-tailed unpaired student's $t$ tests. See also Figure S4

regression analyses demonstrated that virus-specific antibody titers inversely correlated with viral load (Supplementary Figure $4 \mathrm{C}$ ), indicating that the enhanced protection observed in control MBM mice is likely humoral-mediated. Collectively, these data demonstrate that $\mathrm{IL}-10^{+} \mathrm{IL}-21^{+}$co-producing $\mathrm{CD} 4 \mathrm{~T}$ cells are critical for sustaining GC reactions, promoting $\mathrm{B}$ cell 
immunoglobulin class-switching to cytophilic antibody isotypes, and limiting viral replication during chronic LCMV infection.

Tfh-derived IL-10 supports antiviral humoral immunity. Given that IL- $10^{+} \mathrm{IL}-21^{+}$Tfh cells exhibited a more robust GC-like Tfh profile as compared with their IL-10- IL- $21^{+}$counterparts (Fig. 2c-f), we hypothesized that IL-10-producing Tfh cells play a critical role in sustaining GC and B cell-secreted antibody responses during chronic viral infection. To specifically assess the importance of GC Tfh-derived IL-10 in regulating humoral immunity during chronic infection, we generated MBM chimeras in which groups of $\mathrm{CD} 4^{-/-}$mice were lethally irradiated and reconstituted with $\mathrm{BM}$ from the following donors: $C d 4^{-/-}(70 \%)+$ Sh $2 d 1 a^{-/}($Sh $2 d 1 a$ encodes SAP $)(15 \%)+$ either $1 l 10^{-/-}(15 \%)$ or WT (15\%) (Fig. 4a and Supplementary Figure 4D). In this manner, IL-10 deficiency is selectively restricted to GC Tfh cells, but not other CD4 T-cell subsets. These groups of MBM mice were then infected with LCMV Cl13, and on day 21 p.i. we examined the magnitude of the GC and LCMVspecific antibody responses. Notably, abrogation of IL-10 production by Tfh cells resulted in $\sim 2-3$-fold decreases in the proportion and number of $\mathrm{GL}-7^{+} \mathrm{Fas}^{+} \mathrm{GC}$ B cells (Fig. 4b). IL-10 deficiency within the Tfh compartment also had deleterious effects on the Tfh response itself, as MBM chimera mice with $I l 10$ $-/-$ Tfh cells harbored $50 \%$ fewer numbers of CXCR $5^{+} \mathrm{PD}-1^{\text {hiTfh }}$ cells (Supplementary Figure 4E). In alignment with these findings, MBM chimera mice with Tfh-specific IL-10 deletion had 40-50\% lower levels of LCMV-specific IgG1, IgG2a and IgG2b serum antibody titers (Fig. 4c, d), which strongly correlated with their diminished control over viral replication (Fig. 4e and Supplementary Figure 4G). Collectively, these data demonstrate that Tfh-produced IL-10 is essential for maintaining protective humoral responses during chronic viral infection.

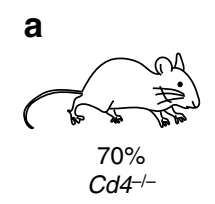

b
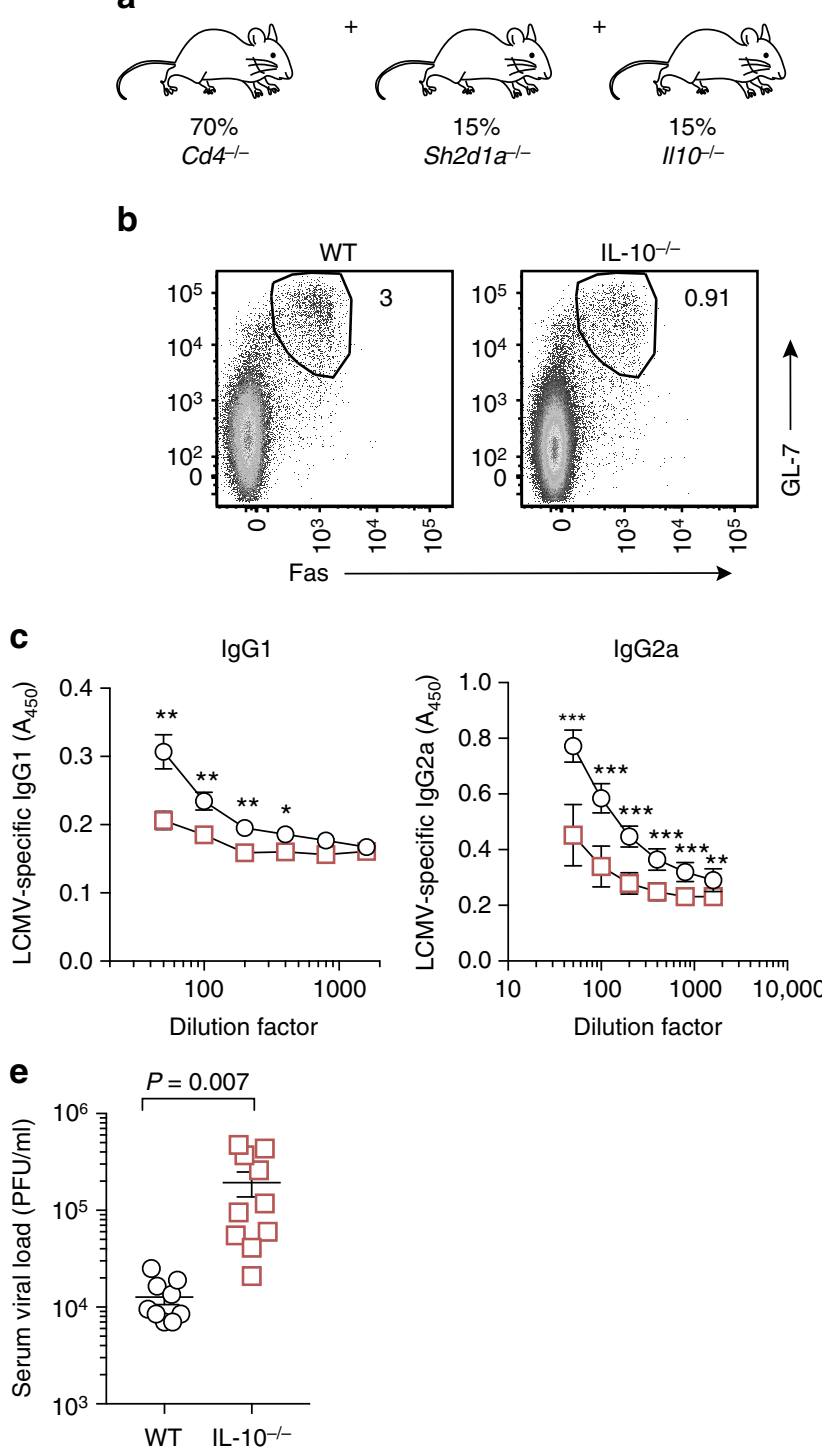
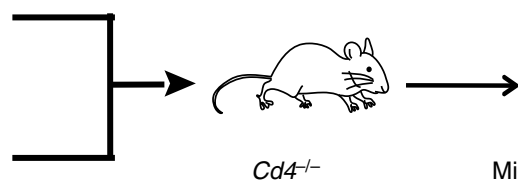

Mice lacking IL-10+ Tfh
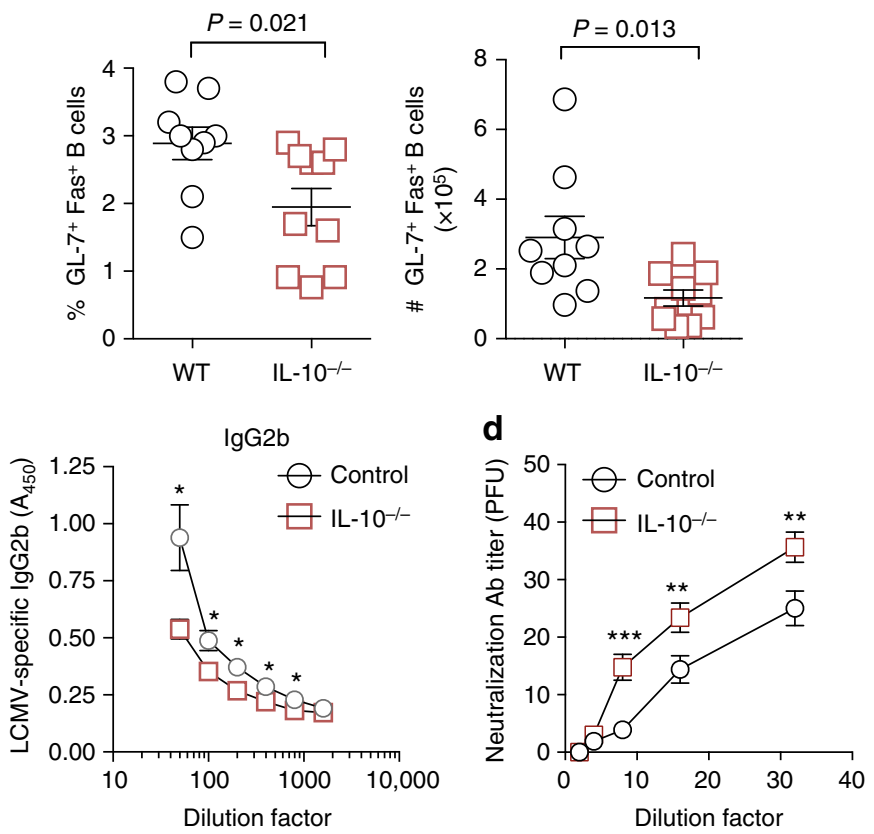

Fig. 4 Tfh-derived IL-10 sustains antiviral humoral immunity. a Experimental scheme of generating MBM chimeric mice with Tfh-specific IL-10 deletion. b-e WT and $1110^{-/-}$Tfh MBM chimeric mice were infected with LCMV Cl13. On day 21 p.i., GC reactions and antibody responses were examined. b Representative flow plots (left) and scatter graphs (right) showing the frequency and total number of GC B cells from WT and I/10-/- Tfh MBM chimeric mice. c Summary graphs showing serum levels of LCMV-specific IgG1, IgG2a, and lgG2b. d Summary graph showing relative antibody neutralization titers for control and $1110^{-/-}$Tfh MBM chimeric mice. e Serum viral titers from WT and $1 / 10^{-/-}$MBM chimeric mice as determined by plaque assay. Summary data (mean +/- SEM in b-e) are pooled from two independent experiments with five mice/group per experiment. ${ }^{\star} P<0.05,{ }^{\star \star} P<0.01,{ }^{\star \star \star} P<0.0001$. Data were analyzed using two-tailed unpaired student's $t$ tests. See also Figure S4 
B cell-intrinsic IL-10R signaling sustains humoral immunity. The necessity of Tfh-derived IL-10 in maintaining humoral immunity during LCMV Cl13 infection raised the question as to whether IL-10 is acting in an intrinsic manner on either Tfh cells themselves, or on B cells to reinforce the GC reaction. To test this, a series of MBM chimeras were generated. First, to test the importance of IL-10 signaling in Tfh cells, Cd4 ${ }^{-/-}$mice were lethally irradiated and reconstituted with BM from the following donors: $C d 4^{-1-}(70 \%)+$ Sh $2 d 1 a^{-/-}(15 \%)+$ either IllOrb $^{-/-}$ (15\%) or WT (15\%) (Supplementary Figure 5A). In this manner, Tfh cells in control mice are capable of responding to IL-10 signaling, whereas Tfh cells in chimeric mice reconstituted with IllOrb $^{-1}$ BM are not. Second, to test whether B cell-intrinsic IL-
$10 \mathrm{R}$ signaling is required to maintain humoral immunity during chronic infection, we reconstituted lethally irradiated WT mice with BM from $\mu M T(70 \%)+$ either Il10rb ${ }^{-1-}(30 \%)$ or WT $(30 \%)$ mice (Fig. 5a). Groups of MBM chimera mice were infected with LCMV Cl13 and on day 21 p.i., we assessed GC- and LCMVspecific antibody responses. Importantly, selective deficiency of IL-10 signaling in Tfh cells had no appreciable effect on either the magnitude of the GC reaction, the level of LCMV-specific antibody titers, or viral containment (Supplementary Figure 5B-E). These data demonstrate that although Tfh-derived IL-10 is essential for sustaining GC and antibody responses, Tfh cells themselves are indirectly affected by IL-10 signaling. In sharp contrast, selective deficiency of IL-10 signaling in B cells severely

a

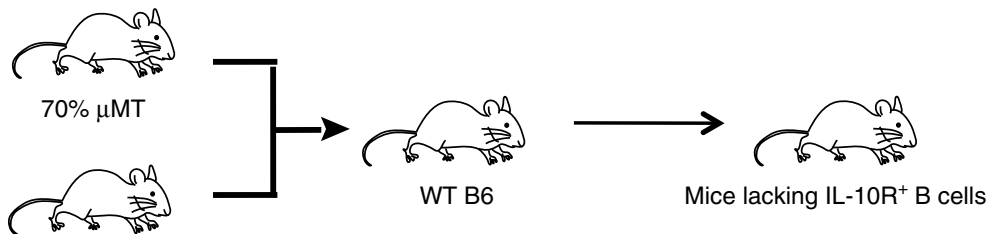

b

$30 \% 1110 \mathrm{rb}^{-1-}$

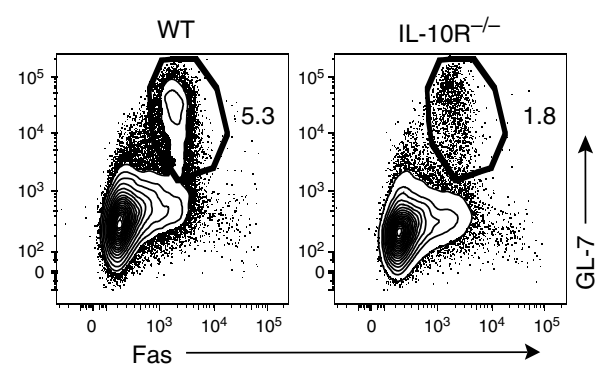

C
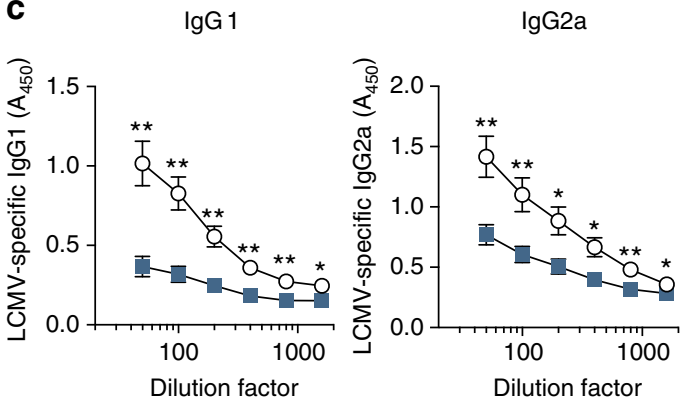

e

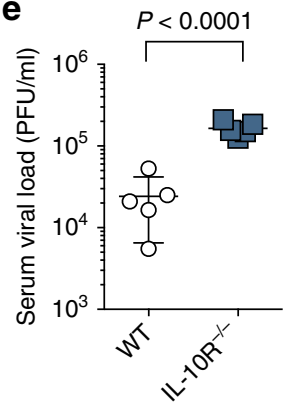

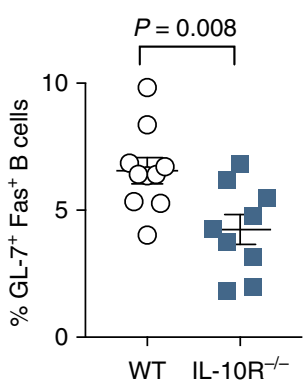
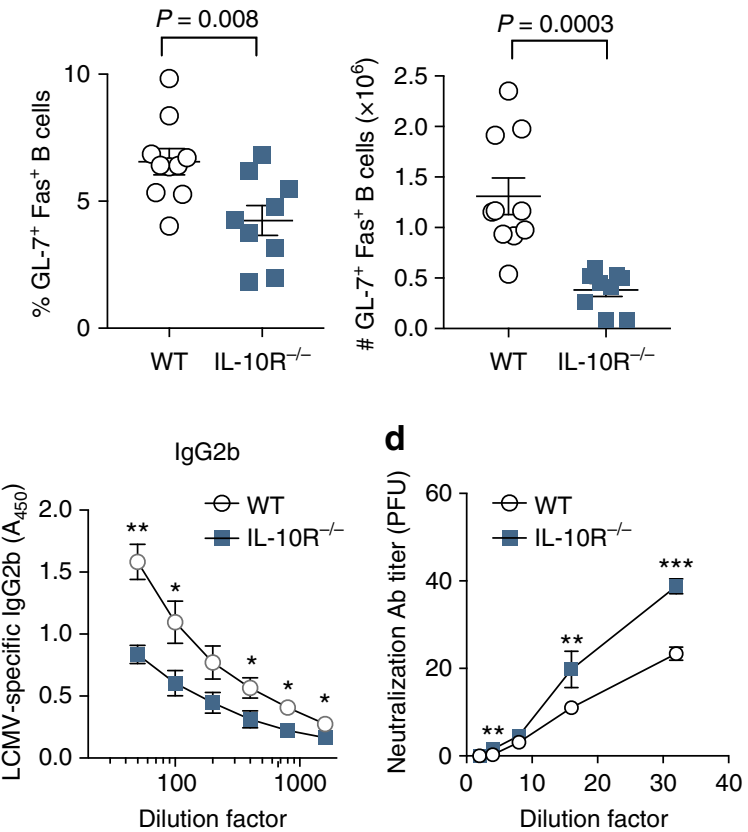

Fig. 5 B cell-intrinsic IL-10 signaling is essential for maintaining GC reactions. a Experimental scheme of generating MBM chimeric mice with B cell-specific II10r deletion. b-d WT and II10r-/- B cell MBM chimeric mice were infected with LCMV Cl13 and B cell responses were examined on day 21 p.i. $\mathbf{b}$ Representative flow plots (left) and scatter graphs (right) showing the frequency and total number of GC B cells from WT and I/10r-/- MBM chimeric mice. c Summary graphs showing serum levels of LCMV-specific IgG1, IgG2a, and IgG2b. d Summary graph showing relative antibody neutralization titers for control and $1110 r^{-/-}$B cell MBM chimeric mice. e Serum viral titers from WT and $1 / 10 r^{-/-}$mice as determined by plaque assay. Summary data (mean $+/$ - SEM in b-e) are pooled from two independent experiments with 4-5 mice/group per experiment. ${ }^{\star} P<0.05,{ }^{\star \star} P<0.01,{ }^{\star \star \star} P<0.0001$. Data were analyzed using two-tailed unpaired student's $t$ tests. See also Figure S6 
impaired the development of GC reactions, as witnessed by two to threefold reductions in the proportion and total numbers of both CXCR5 ${ }^{+}$PD- $1^{\text {hi Tfh cells and GL- }{ }^{+}{ }^{+} \text {Fas }}{ }^{+}$GC B cells (Fig. $5 \mathrm{~b}$ and Supplementary Figure 6A). Moreover, the diminished GC reactions in $1110 \mathrm{rb}^{-1-}$ B cell chimeric mice, were accompanied by $50-60 \%$ reductions in serum titers of LCMV-specific IgG1, IgG2a, and IgG2b isotypes (Fig. 5c, d), and over a 10-fold increase in sera viral titers (Fig. 5e). Additionally, the relative amounts of circulating IgG1, IgG2a, and IgG2b (but not IgM or IgG3 (Supplementary Figure 6B)) once again inversely correlated with viral load (Supplementary Figure 6C), indicating that the protective effects of IL-10 in controlling viral replication is likely antibodymediated. Taken together, these data demonstrate that Tfhderived IL-10 mediates antiviral immunity in a manner that is critically dependent on IL-10 signaling in B cells. Intriguingly, whereas the relative amounts of Bcl- 6 and Blimp-1 in GC B cells did not differ between groups (Supplementary Figure 6D), we identified that expression of T-bet, an important orchestrator of $\mathrm{B}$ cell responses during chronic LCMV infection ${ }^{38}$, was enhanced by $\sim 20 \%$ in GC B cells that retained intact IL-10 signaling (Supplementary Figure 6D).

IL-27 and type I IFNs regulate IL-10 ${ }^{+}$Tfh development. As our results identified a critical role for IL-10-producing Tfh cells in supporting humoral immunity during chronic viral infection (Fig. 4), we next sought to ascertain the inflammatory signals propagated during persistent LCMV infection that contribute to the formation of this protective cell subset. Our Thy1.1 depletion experiments (Fig. 3) suggested that early differentiation signals may be required for the formation of IL- $10^{+} \mathrm{Tfh}$ cells. To further assess whether early antigenic or inflammatory signals are responsible for the induction of $\mathrm{IL}-10^{+} \mathrm{IL}-21^{+} \mathrm{Tfh}$ cells during persistent infection, we transiently depleted CD4 T cells at the time of infection. By day 21 p.i., the proportion of CD $44{ }^{\text {hi }} \mathrm{CD} 4$ $\mathrm{T}$ cells was almost restored to that of control mice (Supplementary Figure 7A), although total numbers of effector T-cells were still reduced $\sim$ twofold (Supplementary Figure 7A). Intriguingly, early depletion of CD4 T cells largely abrogated the generation of IL- $10^{+} \mathrm{IL}-21^{+} \mathrm{Tfh}$ cells despite the formation of $\mathrm{IL}-21^{+}$singlepositive Tfh cells being mostly intact (Supplementary Figure 7B). Moreover, as CD4-depletion results in prolonged viremia ${ }^{39}$, our data indicate that antigenic stimulation alone is not sufficient for the development of IL- $10^{+} \mathrm{IL}-21^{+}$Tfh cells during LCMV Cl13 infection.

One potential explanation for the failure of $\mathrm{IL}-10^{+} \mathrm{IL}-21^{+} \mathrm{Tfh}$ cells to form after CD4-depletion, is that certain viral-induced inflammatory cytokines necessary for the differentiation of $\mathrm{IL}-10^{+} \mathrm{IL}-21^{+} \mathrm{Tfh}$ cells may have rapidly declined at the time CD4 T cells began to reemerge within the host ${ }^{40}$. To test this, we performed in vivo neutralization experiments to block the activity of Tfh-polarizing cytokines IL-6, IL-21, or IL-27, all of which have purported roles in facilitating Tfh differentiation ${ }^{41-44}$. As reported previously, disruption of either IL-6 or IL-21 signaling severely diminished Tfh development (Supplementary Figure 7C). However, we did not observe any consistent differences between groups in the relative proportion of IL-10-producing Tfh cells (Supplementary Figure 7C, D). By contrast, inhibition of the IL-27p28-signaling pathway resulted in $\sim 50 \%$ decreases in the total number of GP66:I-A ${ }^{b}$-specific and effector Tfh cells, and of the Tfh cells that formed, we observed a striking $50 \%$ decrease in the proportion of IL-10-producing cells (Fig. 6a, b and Supplementary Figure 7E, F). Moreover, Tfh expression of Thy1.1 (IL-10) was decreased on a per cell basis by $\sim 25 \%$ when IL-27 was neutralized. These alterations in the Tfh compartment were further associated with reduced serum levels of virus-specific IgG1 and IgG2a (Fig. 6c). Notably, IL-27 has previously been demonstrated to induce IL-10 expression in Th1, Th17, and Tr1 subsets ${ }^{33,45,46}$. To determine whether CD4 intrinsic IL-27 signaling can promote the development of IL- $10^{+} \mathrm{IL}-21^{+} \mathrm{Tfh}$ cells, we performed in vitro culture experiments wherein naive CD4 $\mathrm{T}$ cells were polarized along a Tfh differentiation pathway in the presence of recombinant IL-27. Notably, addition of recombinant IL-27 to the culture simultaneously enhanced the production of IL-10 and IL-21 in in vitro-skewed Tfh cells (Fig. 6d), indicating a potential CD4intrinsic role for IL-27 signaling in promoting the formation of this subset. Collectively, our data demonstrate that IL-27 is a potent inducer of IL-10 expression in Tfh cells during chronic infection (Fig. 2g).

Our finding that IL-27 signaling plays an important role in the formation of IL-10-producing Tfh cells during persistent viral infection, coupled to our observation that IL $10^{+} \mathrm{IL} 21^{+} \mathrm{Tfh}$ cells predominately form during chronic but not acute LCMV infection (Fig. 2), prompted us to investigate whether potential differences in IL-27 availability exists between these two respective infections. To this end, sera levels of IL-27 were assessed at 16 hours, 3 days, and 7 days p.i. in groups of LCMVArm and LCMV Cl13-infected mice. Importantly, although sera levels of IL-27 were similar among experimental groups at 16 hours and 3 days p.i. (Supplementary Figure 7G), we identified over a twofold increase in circulating IL-27 levels in Cl13-infected mice on day 7 p.i. (Fig. 6e), which correspondingly is a time point directly preceding the sharp expansion of IL- $10^{+}$IL- $21^{+}$Tfh cells (Fig. 2b). Taken together, our data suggest that overproduction of IL-27 induced during LCMV Cl13 infection, is one of the potential mechanisms responsible for spurring the development of $\mathrm{IL}-10^{+} \mathrm{IL}-21^{+} \mathrm{Tfh}$ cells.

Finally, persistent LCMV infection is associated with a prolonged type I IFN response ${ }^{47-49}$, which has recently been shown to negatively regulate the induction of protective antibody responses ${ }^{49-51}$. Thus we tested whether blockade of the type I IFN receptor (IFNAR) would enhance the differentiation of IL10-producing Tfh cells. Strikingly, blockade of IFNAR signaling after the first week of infection resulted in approximately a $60 \%$ increase in the proportion of IL-10-producing Tfh cells (Fig. 6f, g and Supplementary Figure $7 \mathrm{H}, \mathrm{I}$ ) which was further associated with a $25 \%$ increase in the proportion of GC B cells on day 21 p.i. (Supplementary Figure 7J). Additionally, IL-10 expression was also increased on a per cell basis by $\sim 30-40 \%$ in GP66:I-A ${ }^{\mathrm{b}}$ specific and effector Tfh cells when IFNAR signaling was abrogated (Fig. 6f, g and Supplementary Figure 7H, I). Collectively, these data demonstrate that the expansion and accumulation of IL- $10^{+}$Tfh cells critically relies on inflammatory and antigenic signals propagated during the early phase of chronic viral infection. Moreover, we identify that IL-27 signaling promotes, while type I IFNs constrain the development of IL-10producing Tfh cells.

\section{Discussion}

In this study, our single-cell transcriptomics analyses identified a subpopulation of IL-10-expressing CD4 T cells that displayed a robust Tfh signature. This finding is rather unexpected, as Th1 and Tr1 cells are implicated as being the predominant sources of IL-10 during chronic viral and parasitic infections ${ }^{4,52-54}$. Importantly, our study demonstrates that a stable population of IL- $10^{+}$Tfh cells develop during chronic but not acute LCMV infection, and that their formation is essential to sustain humoral immunity in the face of persistent antigenic exposure.

While, the transcriptional program and environmental cues important for Tfh differentiation are becoming increasingly welldefined, the precise molecular mechanisms by which Tfh cells sustain humoral immunity during chronic infections is a matter 

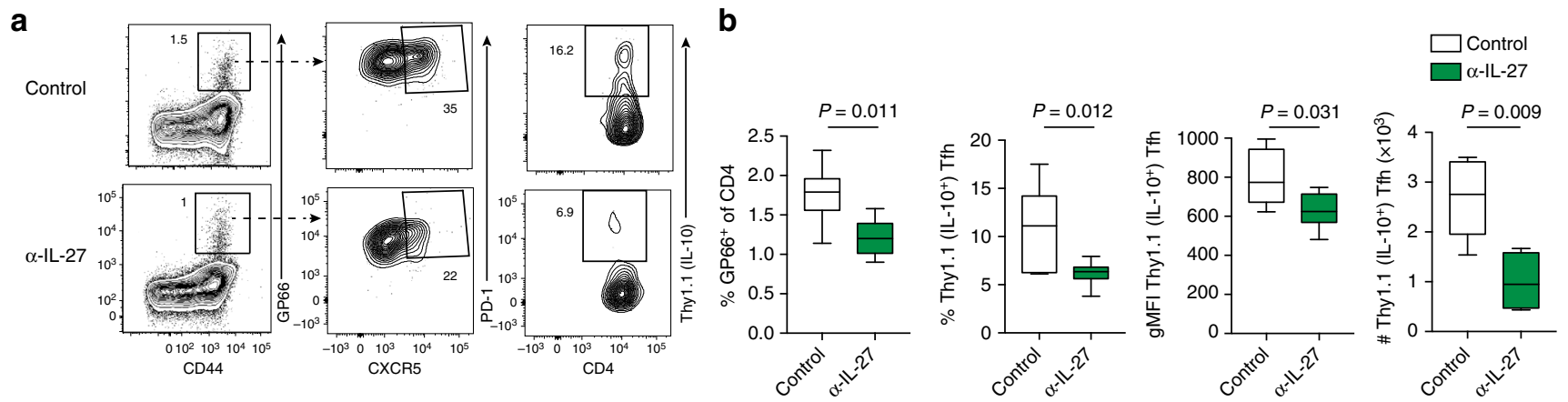

C

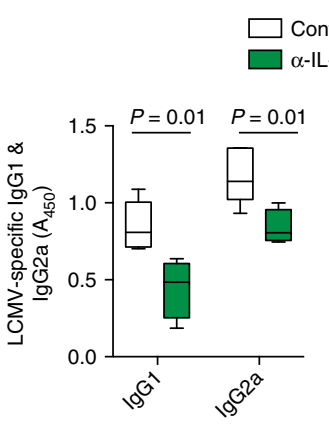

d

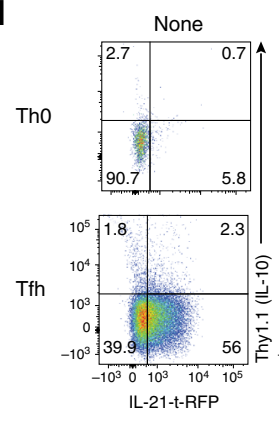

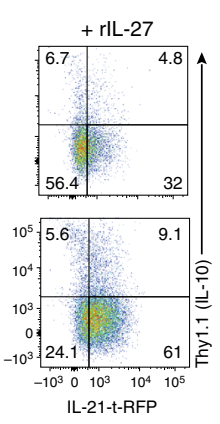

f

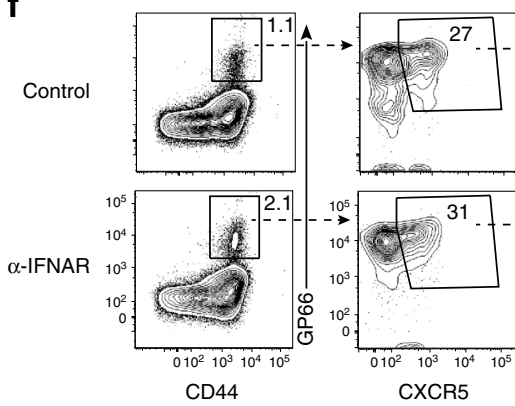

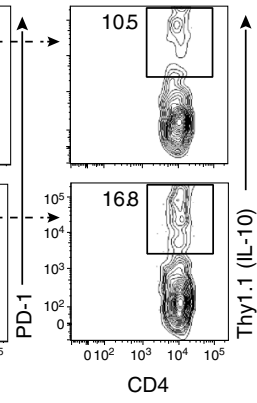

g

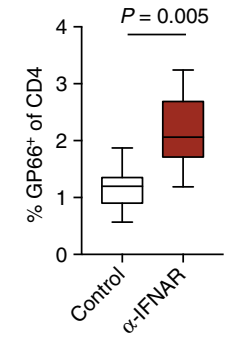

e

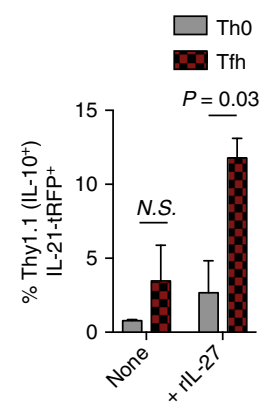

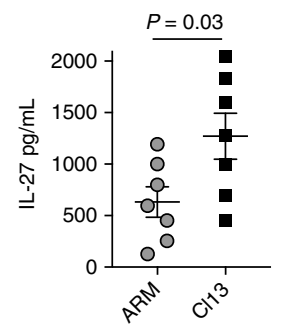
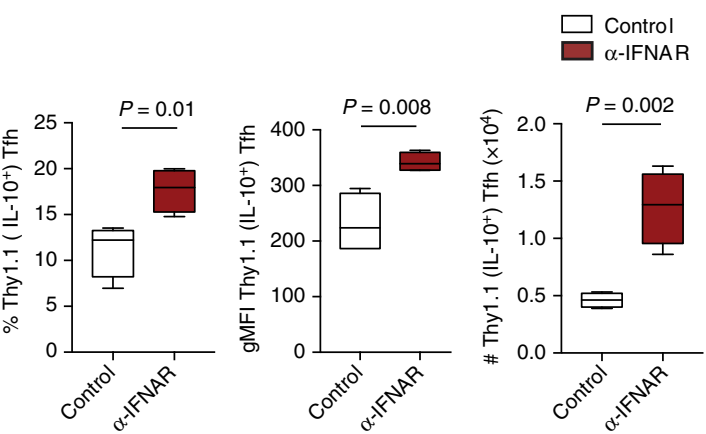

Fig. 6 IL-27 and type I IFNs differentially regulate IL-10+Tfh development. a-c 10BiT-II21-RFP mice were infected with LCMV CI13 and treated with either isotype control or $\alpha-I L-27 p 28$ blocking antibodies on days 3 and 9 p.i. a, b Representative flow plots (a) and summary data (b) depicting the proportion and

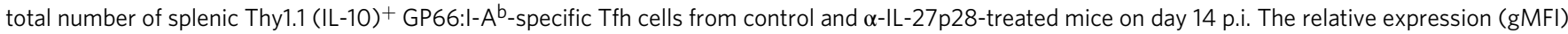
of IL-10 (Thy1.1) in Tfh cells from experimental mice is also depicted in (b). c Summary graph showing serum levels of LCMV-specific lgG1 and IgG2a on day 21 p.i. d Naive CD4 T cells were purified and cultured under Th0 or Tfh-polarizing conditions in the presence or absence of recombinant IL-27. Flow plots (left) and summary data (right) showing the proportion of Thy1.1 (IL-10+) IL-21-tRFP+ cells after 3 days of in vitro culture. e WT mice were infected with LCMV Arm or LCMV Cl13. (e) Summary graph showing serum levels of IL-27 in experimental mice on day 7 p.i. f, $\mathbf{g}$ 10BiT-II21-RFP mice were infected with LCMV CI13 and treated with either isotype control or $\alpha$-IFNAR blocking antibodies on days 10, 14, and 18 p.i. f, $\mathbf{g}$ Representative flow plots (f) and summary data (g) depicting the proportion and total number of splenic Thy1.1 (IL-10) ${ }^{+}$CXCR5 + PD- $1^{+}$GP66:I-A ${ }^{b}$-specific Tfh cells from control and $\alpha$ IFNAR-treated mice on day 21 p.i. The relative expression of IL-10 (Thy1.1) in Tfh cells from experimental mice is also shown in $\mathbf{g}$. Summary data (box and whisker plots showing median (midline) and interquartile range with upper and lower whiskers representing the range of data distribution in $\mathbf{b}$, $\mathbf{c}$, and $\mathbf{g}$ or mean $+/$ - SEM in d, e) are from 3 to 5 mice/group and are representative of two to three independent experiments. ${ }^{\star} P<0.05,{ }^{\star \star} P<0.01,{ }^{\star \star \star} P<0.0001$. Data were analyzed using two-tailed unpaired student's $t$ tests. See also Figure S7

of ongoing investigation. To date, the B cell "helper" effect of IL21 has garnered most of the attention. However, the role of other Tfh-derived cytokines in regulating humoral responses remains less well-explored. In this study, we have identified a unique subpopulation of Tfh cells that co-express IL-10 and IL-21 during persistent infection. Moreover, selective depletion of IL $10^{+} \mathrm{IL}-21^{+} \mathrm{CD} 4 \mathrm{~T}$ cells revealed that the coordinate production of IL-10 and IL-21 by CD4 T cells responding to chronic LCMV infection is essential for sustaining humoral immunity. As T-cellderived IL-10 is generally considered to be an immunosuppressive cytokine that limits viral control $^{4,6-8}$, our finding that
Tfh-derived IL-10 positively instructs GC reactions and antiviral immunity is a rather unexpected discovery.

Notably, our study identifies that distinct environmental and inflammatory signals propagated during chronic but not acute LCMV infection promote the formation of this unique IL- $10^{+} \mathrm{IL}-$ $21^{+}$Tfh subset. Intriguingly, our data also demonstrate that a small population of IL- $10^{+} \mathrm{IL}-21^{+}$Tfh cells also develop during PR8-influenza. Thus, the development of IL- $10^{+} \mathrm{IL}-21^{+}$Tfh cells appears to be context-dependent, and it is possible that these cells may play a role in regulating humoral responses during various immunological settings. Importantly, however, our data 
additionally indicate that the formation of IL- $10^{+} \mathrm{IL}-21^{+} \mathrm{Tfh}$ cells during influenza appears to be rather transient. Moreover, our unpublished observations indicate that the majority of IL-10producing $\mathrm{CD} 4 \mathrm{~T}$ cells with a $\mathrm{CXCR} 5^{+} \mathrm{PD}-1^{+}$phenotype coexpress Foxp3 during PR8 infection, suggesting that this minor IL- $10^{+}$subset may be predominantly comprised of TFR cells. This observation is consistent with two recently published reports identifying that TFR cells significantly expand after viral clearance, including both PR8 influenza and LCMV-Arm infections ${ }^{55,56}$. While the expansion of TFR cells during PR8 was found to be essential to prevent autoreactive B cell responses ${ }^{55}$, this latter study unexpectedly demonstrated that TFR-derived IL10 can actually promote GC B cell differentiation ${ }^{56}$. However, whether TFR-derived IL-10 has any impact on B cell-secreted antibody responses or viral control remains unclear.

By contrast, our study demonstrates that only a small proportion $(<15 \%)$ of $\mathrm{IL}-10^{+} \mathrm{CXCR}^{+} \mathrm{PD}-1^{+} \mathrm{CD} 44^{\mathrm{hi}} \mathrm{CD} 4 \mathrm{~T}$ cells coexpress Foxp3 during chronic LCMV infection, and that Foxp3 expression is undetectable in the GP66-specific Tfh compartment, indicating the absence of the TFR lineage among virus-specific CD4 T cells. This observation is in agreement with a previous report that identified that GC Tfh and TFR subsets are generated from distinct TCR repertories, with Tfh cells expressing antigenresponsive TCRs to promote antibody responses whereas TFRs appear to express potentially autoreactive TCRs in order to suppress autoimmunity ${ }^{57}$. Our data additionally demonstrate that IL-21 is not highly expressed in Foxp $3^{+}$TFR cells during LCMV Cl13 infection, further indicating that $\mathrm{IL}-10^{+} \mathrm{IL}-21^{+} \mathrm{Tfh}$ cells are distinct from the TFR lineage.

Given our findings that Tfh-derived IL-10 is essential for promoting antiviral humoral immunity, it was of interest to determine the molecular signals required for the development of $\mathrm{IL}-10^{+} \mathrm{Tfh}$ cells. Our IL- $10^{+} \mathrm{IL}-21^{+} \mathrm{CD} 4$-depletion experiments indicate that early inflammatory signals propagated during the first few weeks of LCMV Cl13 infection are necessary for the formation of IL- $10^{+} \mathrm{IL}-21^{+} \mathrm{Tfh}$ cells. In alignment with this idea, the Tfh-skewing cytokine IL-6 is reportedly produced in a biphasic fashion ${ }^{40}$. Thus, particular cytokines important for Tfh differentiation may have been absent at the time $\mathrm{CD} 4 \mathrm{~T}$ cells began to re-emerge within the host following transient depletion of either $\mathrm{CD} 4$ or $\mathrm{IL}-10^{+} \mathrm{IL}-21^{+} \mathrm{CD} 4 \mathrm{~T}$ cells. Therefore, we explored a potential role for the cytokines IL-6, IL-21, and IL-27 in the early induction of IL-10-producing Tfh cells during LCMV Cl13 infection. Our in vivo neutralization experiments suggest that IL- 6 and IL-21 largely impact the magnitude of the Tfh response, whereas IL-27 appears to be one of the key cytokines involved in the induction of $\mathrm{IL}-10^{+} \mathrm{Tfh}$ cells during chronic infection. However, it remains to be determined whether other cytokines, such as IL-12 or IFN- $\gamma$, both of which have purported roles in promoting Tfh differentiation ${ }^{58-60}$, further contribute to the development of IL- $10^{+} \mathrm{Tfh}$ cells. Intriguingly, our in vitro $\mathrm{Tfh}$ polarization experiments indicate that direct IL-27 signaling may promote the development of IL- $10^{+} \mathrm{Tfh}$ cells, although it remains unclear as to whether this induction process can occur in vivo. Additionally, although the pathways downstream of IL27R signaling that promote the differentiation of IL- $10^{+} \mathrm{Tfh}$ cells remain relatively unexplored, our RNA-seq analyses potentially indicate that c-Maf, a known downstream target of IL-27 signaling ${ }^{33}$, may be associated with this induction. Notably, several studies have identified that c-Maf plays a critical role in facilitating Tfh differentiation ${ }^{61,62}$ as well as in promoting IL-10 expression in various CD4 T-cell populations ${ }^{32,63}$. Collectively, our data indicate that IL-27 appears to be one of the important cytokines in promoting the formation of IL- $10^{+}$Tfh cells during LCMV Cl13 infection, although it is possible that other cytokines may also contribute to the development of this protective subset.

Intriguingly, our study has also identified that type I IFNs suppress the formation of IL- $10^{+} \mathrm{Tfh}$ cells during chronic infection. Several recent studies have demonstrated an inhibitory role for type I IFNs in the development of neutralizing antibody responses during persistent viral infection ${ }^{49-51}$. In these scenarios, the inhibitory effects of type I IFNs occurred in a B cellextrinsic fashion and involved IFNAR signaling in multiple cell types, including myeloid cells, dendritic cells, and $\mathrm{T}$ cells. Interestingly, previous studies have also identified that IFNAR blockade during chronic infection results in enhanced viral clearance $^{47,48}$. Our study further expands on these studies and demonstrates that type I IFNs may also impede the development of $\mathrm{IL}-10^{+} \mathrm{Tfh}$ cells. Hence, our results may also indicate a potential mechanism by which type I IFNs function to impede the development of protective antibody responses during persistent infection.

Collectively, our work highlights a previously unrecognized circuit wherein Tfh-secreted IL-10 maintains GC reactions and supports the production of virus-specific antibodies. Importantly, our study also identifies that B cell-intrinsic IL-10 signaling is pivotal for sustaining humoral responses during chronic viral infection. This finding is consistent with a recent report that demonstrated IL-10R-signaling in B cells is essential for the induction of GC reactions and humoral immunity during Plasmodium infection ${ }^{64}$. Thus, the necessity of B cell-intrinsic IL-10R signaling in sustaining humoral immunity may be a generalizable feature of chronic infections. In summary, our study ascertains that Tfh-derived IL-10 is a key positive regulator of GC reactions and antibody responses during chronic infection. Thus, therapeutic strategies aimed at enhancing Tfh production of IL-10 may serve to bolster humoral immunity during persistent viral infection.

\section{Methods}

Mice and viruses. All mice were bred and maintained in a closed breeding facility, and mouse handling conformed to the requirements of the Institutional Animal Care and Use Guidelines of Medical College of Wisconsin. C57BL/6, Il10r ${ }^{-1-}$, Il10 $-/-, I l 21^{-/-}$, and $C d 4^{-/-}$mice were purchased from Jackson Laboratory (Bar Harbor, ME). IL-10 and IL-21 double-reporter mice were generated by crossbreeding IL-21tRFP mice ${ }^{12,25}$ with ten BiT mice (kindly provided by Dr. Casey Weaver, University of Alabama at Birmingham, AL). All mice used in accordance with animal protocols approved by the Medical College of Wisconsin Institutional Animal Care and Use Committee. LCMV Armstrong (Arm) was intraperitoneally injected into mice $\left(2 \times 10^{5} \mathrm{PFU} /\right.$ mouse $)$ to establish an acute infection. LCMV clone 13 (Cl13) was intravenously injected into mice $\left(2 \times 10^{6} \mathrm{PFU} / \mathrm{mouse}\right)$ to establish chronic infection. Both strains of viruses were prepared by a single passage on BHK21 cells, and viral titers were determined by plaque formation assay on Vero cells.

Flow cytometry. Mouse splenocytes were harvested, subjected to red blood cell lysis, washed, and stained as previously described ${ }^{12}$. Staining with GP66:A-b PE tetramer (NIH tetramer core facility) was performed at room temperature for $1 \mathrm{~h}$. Transcription factor staining was performed using a Foxp3 staining buffer set (eBioscience, CA). To detect Tfh cells, CXCR5 was stained for using rat anti-mouse CXCR5 antibody (BD Bioscience, CA), followed by incubation of a secondary biotin-SP-conjugated Affinipure $\mathrm{F}\left(\mathrm{Ab}^{\prime}\right) 2$ goat anti-rat IgG (Jackson Immunoresearch, PA). At the last step, cells were stained with streptavidin-APC (Invitrogen, CA) together with other surface markers. In some experiments, anti-CXCR5 APC or APCcy7 antibody conjugates were used. All antibodies used in this study are listed in Supplemental Table 1. All flow cytometry data were acquired on an LSRII (BD Biosciences, CA) and analyzed by FlowJo (Treestar, OR).

In vitro CD4 polarization assays. Twenty-four-well plates were coated with antiCD3 antibodies (2 ug/mL; Biolegend) for $2 \mathrm{~h}$ at 37 degrees. Naive CD4 T cells from 10Bit $I L-21$-tRFP reporter mice were purified using the EasySep CD4 T cell 
isolation kit from Stemcell technologies, and $1 \times 10^{6}$ cells were plated per well. Cells were stimulated for 3 days at 37 degrees in the presence of anti-CD28 antibodies (2 $\mu \mathrm{g} / \mathrm{ml}$; Biolegend). Th0 cells were cultured in the presence of $20 \mathrm{ng} / \mathrm{mL}$ IL-2. Tfh polarization was achieved by adding $50 \mathrm{ng} / \mathrm{mL}$ recombinant IL- 6 and anti-IFN- $\gamma$ and anti-IL-4-neutralizing antibodies $(1 \mu \mathrm{g} / \mathrm{mL}$ each; Biolegend). Some of the Th0 and Tfh-polarized cells also received recombinant IL-27 $(25 \mathrm{ng} / \mathrm{mL})$ at the start of the culture. After 3 days of culture, cells were stimulated with PMA and Ionomycin for $1 \mathrm{~h}$, at which point monensin was added to the culture wells, and cells were then stimulated for an additional $4 \mathrm{~h}$. Polarized CD4 T cells were then assessed for Thyl.1 (IL-10) and IL-21-tRFP expression by flow cytometry.

Mouse cytokine assay. Serum samples were obtained from LCMV Armstrong and LCMV Cl13-infected WT mice at $16 \mathrm{~h}, 3$ days, and 7 days post infection. Serum samples were sent to Eve Technologies (Calgary, AB), and analyzed using a multiplex cytokine array.

ELISA for LCMV-specific antibody. To quantify LCMV-specific antibodies, the Cl13-infected BHK cell lysates were used to coat the plate overnight and blocked with $3 \% \mathrm{BSA} / \mathrm{PBS} / 0.05 \%$ Tween $20^{1}$. After $1 \mathrm{~h}$ of blocking, sera from infected mice were serially diluted and incubated on the plate for $90 \mathrm{~min}$. Subsequently antibody detection was performed for IgM, IgG1, IgG2a, IgG2b, and IgG3 isotypes using HRP conjugated anti-mouse antibodies (Southern Biotechnologies, AL) and relative absorption was measured.

LCMV neutralization assay. Detection of neutralizing activity against LCMV in mouse sera was determined using a focus-forming assay, according to a previously published protocol ${ }^{65}$. In brief, diluted sera were UV irradiated and incubated with $60 \mathrm{PFU}$ of LCMV-CL13 for $90 \mathrm{~min}$ at $37^{\circ} \mathrm{C}$. Then MC57G mouse fibroblast cells were added to each well and incubated for 2 to $3 \mathrm{~h}$. Thereafter, $1 \%$ methylcellulose was added, and the cells were placed in the incubator. After $48 \mathrm{~h}$ of incubation, cells were fixed with $4 \%$ formaldehyde in PBS followed by permeabilization with $1 \%$ Triton X-100. Foci were visualized by staining with the anti-LCMV nucleoprotein antibody (VL-4, Bio X Cell, NH).

Administration of biologics. To deplete $\mathrm{CD} 4 \mathrm{~T}$ cells, mice received i.p. injections of $500 \mu \mathrm{g}$ anti-CD4 antibody (clone GK1.5 from BioXCell, NH) or IgG2a isotype control 1 day before LCMV Cl13 infection and on the two consecutive days following infection. Anti-mouse Thy1.1 (clone 19E12 from BioXCell, NH) antibodies were administered on days 4 and 6 post infection to deplete Thyl.1-expressing cells. In all cases, the depletion efficacy was confirmed via flow cytometry. For IL27 neutralization experiments, mice were treated on days 3 and 9 p.i. with either $500 \mu \mathrm{g}$ IgG2a isotype control or $500 \mu \mathrm{g}$ anti-IL-27p28 blocking antibodies (MM27.7B1; as previously described ${ }^{66}$. For in vivo blockade of type I IFN signaling, mice were treated with either $500 \mu \mathrm{g}$ of MOPC isotype control (BioXcell) or $500 \mu \mathrm{g}$ of anti-IFNAR (MAR1-5A3; (BioXcell)) blocking antibodies on days 10, 14, and 18 p.i.

Mixed bone marrow (MBM) chimeras. For MBM chimera experiments, recipient mice were irradiated with 6.5 and 5.5 Gy separated by $8 \mathrm{~h}$. Bone marrow from various donor mice (as depicted in Figures) were mixed at the indicated ratios, and a total of $\sim 6 \times 10^{6}$ cells were transferred i.v. Mice were maintained on oral sulfamethoxazole for 2 weeks. Chimerism was assessed at 7 weeks in peripheral blood using congenic markers. Chimerism in the $\mathrm{CD} 4 \mathrm{~T}$ cell compartment in mice reconstituted with bone marrow from $C d 4^{-/-}$mice, Sh2 $d 1 a^{-/-}$mice, and either Illo $0^{-/-}$or WT mice was $~ 55 \%$ Sh $2 \mathrm{~d} \mathrm{a}^{-/-}$and $45 \%$ either $I l 10^{-/-}$or WT amongst 20 experimental mice in two independent experiments. Chimerism in the B cell compartment in mice reconstituted with bone marrow from $\mu M T$ mice + bone marrow from either Il10rb- ${ }^{-1-}$ or WT mice was greater than $93 \%$. Experimental MBM mice were infected with LCMV Cl13 at 8 weeks post reconstitution.

Single-cell RNA sequencing. Thy1.1 $1^{+}\left(\mathrm{IL}-10^{+}\right) \mathrm{GP}_{61-80}$-specific CD4 T cells were FACS-sorted from LCMV Cl13-infected mice on day 16 p.i. and were loaded on the Chromium Controller (10x Genomics). Single-cell RNA-seq libraries were prepared using the Chromium Single Cell 3' v2 Reagent Kit (10x Genomics) according to manufacturer's protocol. Libraries were loaded onto an Illumina NextSeq with the NextSeq 500/550 High Output Kit v2 (150 cycles) (FC-404-2002, Illumina) with the following conditions: 26 cycles for read 1, 98 cycles for read 2 , and 8 cycles for i7 index. Python Run Downloader (Illumina) was used to download raw sequencing data. Cell Ranger (10x Genomics) functions mkfastq and count were used to demultiplex the sequencing data and generate gene-barcode matrices, respectively. All scRNA-seq analysis was performed in R (version 3.4.0) using the package Seurat (version 2.2.0) ${ }^{67}$. Number of genes detected per cell, number of UMIs, and percent mitochondrial genes were plotted, and outliers were removed (number of genes over 2500, number of UMIs over 8000, and percent mitochondrial genes over 0.08) to filter out doublets and dead cells, leaving 677 of the original 691 cells. Cell cycle genes were regressed out using a list of S phase genes and G2/M phase genes ${ }^{67,68}$. Principal component analysis was performed, and the top three most statistically significant principal components were used for $t$-SNE analysis, with 2000 iterations and a perplexity parameter of 30 .

Bulk RNA sequencing. Naive $\left(\mathrm{CD} 44^{10}\right) \mathrm{CD} 4 \mathrm{~T}$ cells, $\mathrm{IL}-21^{+}$single-positive Tfh cells $\left(\mathrm{CD} 44^{\mathrm{hi}} \mathrm{CXCR}^{+} \mathrm{PD}-\mathrm{1}^{\mathrm{hi}}\right)$, and $\mathrm{IL}-10^{+} \mathrm{IL}-21^{+}$double-positive Tfh cells were FACS-sorted from the spleens of LCMV Cl13-infected 10BiT-Il21tRFP doublereporter mice at day 18 p.i. RNA-seq libraries were prepared using SMART-seq technology ${ }^{69}$ and sequenced on an Illumina NextSeq. Reads were aligned to mm9 transcriptome using TopHat (version 2.1.1) and differential gene expression was determined using Cuffdiff (Cufflinks version 2.2.2) ${ }^{70}$.

Microscopy. Spleens were fixed with periodate-lysine paraformaldahyde and then snap-frozen in OCT tissue-freezing solution and stored at $-80^{\circ} \mathrm{C}$. Tissues were cut into $7-\mu \mathrm{m}$ sections, placed onto superfrost glass slides and stored at $-80^{\circ} \mathrm{C}$ until staining. Prior to immunostaining, tissues were rehydrated and blocked with $2 \%$ BSA, $5 \%$ goat serum, and $2 \%$ fetal calf serum. Reagents used to stain sections are listed in Supplementary Table 1. Images were obtained using a Nikon TI2-E inverted microscope at 40x magnification and Imaris software (Bitplane) version 9 was used to prepare images.

Statistical analyses. Statistical tests were performed using Graphpad Prism 7. Pvalues were calculated using two-tailed unpaired student's $t$ tests, unless otherwise indicated.

\section{Data availability}

The bulk RNA-seq and single-cell RNA-seq data have been deposited in the GEO database with the accession code GSE111027. A reporting summary for this Article is available as a Supplementary Information file. All other relevant raw data are available from the corresponding author upon request.

Received: 17 July 2018 Accepted: 31 October 2018

Published online: 28 November 2018

\section{References}

1. Fahey, L. M. et al. Viral persistence redirects $\mathrm{CD} 4 \mathrm{~T}$ cell differentiation toward T follicular helper cells. J. Exp. Med 208, 987-999 (2011).

2. Wherry, E. J. T cell exhaustion. Nat. Immunol. 12, 492-499 (2011).

3. Crawford, A. et al. Molecular and transcriptional basis of CD4 $(+) \mathrm{T}$ cell dysfunction during chronic infection. Immunity 40, 289-302 (2014)

4. Parish, I. A. et al. Chronic viral infection promotes sustained Th1-derived immunoregulatory IL-10 via BLIMP-1. J. Clin. Invest 124, 3455-3468 (2014).

5. Elsaesser, H., Sauer, K. \& Brooks, D. G. IL-21 is required to control chronic viral infection. Science 324, 1569-1572 (2009).

6. Brooks, D. G. et al. IL-10 and PD-L1 operate through distinct pathways to suppress T-cell activity during persistent viral infection. Proc. Natl. Acad. Sci. USA 105, 20428-20433 (2008).

7. Brooks, D. G., Walsh, K. B., Elsaesser, H. \& Oldstone, M. B. IL-10 directly suppresses $\mathrm{CD} 4$ but not $\mathrm{CD} 8 \mathrm{~T}$ cell effector and memory responses following acute viral infection. Proc. Natl. Acad. Sci. USA 107, 3018-3023 (2010).

8. Ejrnaes, M. et al. Resolution of a chronic viral infection after interleukin-10 receptor blockade. J. Exp. Med 203, 2461-2472 (2006).

9. Saraiva, M. \& O'Garra, A. The regulation of IL-10 production by immune cells. Nat. Rev. Immunol. 10, 170-181 (2010).

10. Laidlaw, B. J. et al. Production of IL-10 by CD4 $(+)$ regulatory T cells during the resolution of infection promotes the maturation of memory $\mathrm{CD} 8(+)$ T cells. Nat. Immunol. 16, 871-879 (2015).

11. Wilson, E. B. \& Brooks, D. G. The role of IL-10 in regulating immunity to persistent viral infections. Curr. Top. Microbiol Immunol. 350, 39-65 (2011).

12. Xin, G. et al. A critical role of IL-21-Induced BATF in sustaining CD8-T-cellmediated chronic viral control. Cell Rep. 13, 1118-1124 (2015).

13. Yi, J. S., Du, M. \& Zajac, A. J. A vital role for interleukin-21 in the control of a chronic viral infection. Science 324, 1572-1576 (2009).

14. Frohlich, A. et al. IL-21R on T cells is critical for sustained functionality and control of chronic viral infection. Science 324, 1576-1580 (2009).

15. Kuchen, S. et al. Essential role of IL-21 in B cell activation, expansion, and plasma cell generation during $\mathrm{CD} 4+\mathrm{T}$ cell-B cell collaboration. J. Immunol. 179, 5886-5896 (2007).

16. Crotty, S. Follicular helper CD4 T cells (TFH). Annu Rev. Immunol. 29, 621-663 (2011). 
17. Kurosaki, T., Kometani, K. \& Ise, W. Memory B cells. Nat. Rev. Immunol. 15, 149-159 (2015).

18. Nurieva, R. I. et al. Bcl6 mediates the development of T follicular helper cells. Science 325, 1001-1005 (2009).

19. Gitlin, A. D., Shulman, Z. \& Nussenzweig, M. C. Clonal selection in the germinal centre by regulated proliferation and hypermutation. Nature 509, 637-640 (2014).

20. Johnston, R. J. et al. Bcl6 and Blimp-1 are reciprocal and antagonistic regulators of T follicular helper cell differentiation. Science 325, 1006-1010 (2009).

21. Choi, Y. S. et al. ICOS receptor instructs T follicular helper cell versus effector cell differentiation via induction of the transcriptional repressor Bcl6. Immunity 34, 932-946 (2011).

22. Akiba, H. et al. The role of ICOS in the CXCR5 + follicular B helper T cell maintenance in vivo. J. Immunol. 175, 2340-2348 (2005).

23. Qi, H., Cannons, J. L., Klauschen, F., Schwartzberg, P. L. \& Germain, R. N. SAP-controlled T-B cell interactions underlie germinal centre formation. Nature 455, 764-769 (2008).

24. Yusuf, I. et al. Germinal center T follicular helper cell IL-4 production is dependent on signaling lymphocytic activation molecule receptor (CD150). J. Immunol. 185, 190-202 (2010).

25. Weinstein, J. S. et al. TFH cells progressively differentiate to regulate the germinal center response. Nat. Immunol. 17, 1197-1205 (2016).

26. Luthje, K. et al. The development and fate of follicular helper T cells defined by an IL-21 reporter mouse. Nat. Immunol. 13, 491-498 (2012).

27. Sahoo, A. et al. Batf is important for IL-4 expression in T follicular helper cells. Nat. Commun. 6, 7997 (2015).

28. Hirota, K. et al. Plasticity of Th17 cells in Peyer's patches is responsible for the induction of T cell-dependent IgA responses. Nat. Immunol. 14, 372-379 (2013).

29. Wang, Y. et al. Germinal-center development of memory B cells driven by IL9 from follicular helper T cells. Nat. Immunol. 18, 921-930 (2017).

30. Maynard, C. L. et al. Regulatory T cells expressing interleukin 10 develop from Foxp3 + and Foxp3- precursor cells in the absence of interleukin 10. Nat. Immunol. 8, 931-941 (2007).

31. Sun, J., Dodd, H., Moser, E. K., Sharma, R. \& Braciale, T. J. CD4 + T cell help and innate-derived IL-27 induce Blimp-1-dependent IL-10 production by antiviral CTLs. Nat. Immunol. 12, 327-334 (2011).

32. Xu, J. et al. c-Maf regulates IL-10 expression during Th17 polarization. J. Immunol. 182, 6226-6236 (2009).

33. Pot, C. et al. Cutting edge: IL-27 induces the transcription factor c-Maf, cytokine IL-21, and the costimulatory receptor ICOS that coordinately act together to promote differentiation of IL-10-producing $\operatorname{Tr} 1$ cells. J. Immunol. 183, 797-801 (2009).

34. Shulman, Z. et al. Dynamic signaling by $\mathrm{T}$ follicular helper cells during germinal center B cell selection. Science 345, 1058-1062 (2014).

35. Johnston, R. J., Choi, Y. S., Diamond, J. A., Yang, J. A. \& Crotty, S. STAT5 is a potent negative regulator of TFH cell differentiation. J. Exp. Med. 209, 243-250 (2012).

36. Sage, P. T. et al. Suppression by TFR cells leads to durable and selective inhibition of B cell effector function. Nat. Immunol. 17, 1436-1446 (2016).

37. Linterman, M. A. et al. Foxp3 + follicular regulatory T cells control the germinal center response. Nat. Med 17, 975-982 (2011).

38. Barnett, B. E. et al. Cutting Edge: B Cell-Intrinsic T-bet Expression Is Required To Control Chronic Viral Infection. J. Immunol. 197, 1017-1022 (2016).

39. Battegay, M. et al. Enhanced establishment of a virus carrier state in adult CD4 + T-cell-deficient mice. J. Virol. 68, 4700-4704 (1994)

40. Harker, J. A., Lewis, G. M., Mack, L. \& Zuniga, E. I. Late interleukin-6 escalates $\mathrm{T}$ follicular helper cell responses and controls a chronic viral infection. Science 334, 825-829 (2011).

41. Choi, Y. S. et al. Bcl6 expressing follicular helper CD4 T cells are fate committed early and have the capacity to form memory. J. Immunol. 190, 4014-4026 (2013).

42. Eto, D. et al. IL-21 and IL-6 are critical for different aspects of B cell immunity and redundantly induce optimal follicular helper $\mathrm{CD} 4 \mathrm{~T}$ cell (Tfh) differentiation. PLoS One 6, e17739 (2011).

43. Nurieva, R. I. et al. Generation of $\mathrm{T}$ follicular helper cells is mediated by interleukin-21 but independent of T helper 1, 2, or 17 cell lineages. Immunity 29, 138-149 (2008)

44. Batten, M. et al. IL-27 supports germinal center function by enhancing IL-21 production and the function of T follicular helper cells. J. Exp. Med. 207, 2895-2906 (2010).

45. Murugaiyan, G. et al. IL-27 is a key regulator of IL-10 and IL-17 production by human CD4 + T cells. J. Immunol. 183, 2435-2443 (2009).

46. Batten, M. et al. Cutting edge: IL-27 is a potent inducer of IL-10 but not FoxP3 in murine T cells. J. Immunol. 180, 2752-2756 (2008).

47. Wilson, E. B. et al. Blockade of chronic type I interferon signaling to control persistent LCMV infection. Science 340, 202-207 (2013).
48. Teijaro, J. R. et al. Persistent LCMV infection is controlled by blockade of type I interferon signaling. Science 340, 207-211 (2013).

49. Moseman, E. A., Wu, T., de la Torre, J. C., Schwartzberg, P. L. \& McGavern, D. B. Type I interferon suppresses virus-specific B cell responses by modulating CD8 + T cell differentiation. Sci. Immunol. 1, eaah3565 (2016).

50. Fallet, B. et al. Interferon-driven deletion of antiviral B cells at the onset of chronic infection. Sci. Immunol. 1, eaah6817 (2016)

51. Sammicheli, S. et al. Inflammatory monocytes hinder antiviral B cell responses. Sci. Immunol. 1, eaah6789 (2016).

52. Montes de Oca, M. et al. Blimp-1-dependent IL-10 production by Tr1 cells regulates TNF-mediated tissue pathology. PLoS Pathog. 12, e1005398 (2016).

53. Lonnberg, T. et al. Single-cell RNA-seq and computational analysis using temporal mixture modelling resolves Th1/Tfh fate bifurcation in malaria. Sci. Immunol. 2, eaal2192 (2017).

54. Freitas do Rosario, A. P. et al. IL-27 promotes IL-10 production by effector Th1 CD4 + T cells: a critical mechanism for protection from severe immunopathology during malaria infection. J. Immunol. 188, 1178-1190 (2012).

55. Botta, D. et al. Dynamic regulation of $\mathrm{T}$ follicular regulatory cell responses by interleukin 2 during influenza infection. Nat. Immunol. 18, 1249-1260 (2017).

56. Laidlaw, B. J. et al. Interleukin-10 from $\mathrm{CD} 4(+)$ follicular regulatory T cells promotes the germinal center response. Sci. Immunol. 2, eaan0368 (2017).

57. Maceiras, A. R. et al. T follicular helper and $\mathrm{T}$ follicular regulatory cells have different TCR specificity. Nat. Commun. 8, 15067 (2017).

58. Weinstein, J. S. et al. STAT4 and T-bet control follicular helper T cell development in viral infections. J. Exp. Med 215, 337-355 (2018).

59. Schmitt, N. et al. Human dendritic cells induce the differentiation of interleukin-21-producing $\mathrm{T}$ follicular helper-like cells through interleukin-12. Immunity 31, 158-169 (2009).

60. Lee, S. K. et al. Interferon-gamma excess leads to pathogenic accumulation of follicular helper T cells and germinal centers. Immunity 37, 880-892 (2012).

61. Andris, F. et al. The Transcription factor c-Maf promotes the differentiation of follicular helper T cells. Front Immunol. 8, 480 (2017).

62. Kroenke, M. A. et al. Bcl6 and Maf cooperate to instruct human follicular helper CD4 T cell differentiation. J. Immunol. 188, 3734-3744 (2012).

63. Apetoh, L. et al. The aryl hydrocarbon receptor interacts with c-Maf to promote the differentiation of type 1 regulatory T cells induced by IL-27. Nat. Immunol. 11, 854-861 (2010).

64. Guthmiller, J. J., Graham, A. C., Zander, R. A., Pope, R. L. \& Butler, N. S. Cutting edge: IL-10 is essential for the generation of germinal center B cell responses and anti-plasmodium humoral immunity. J. Immunol. 198, 617-622 (2017).

65. Battegay, M. et al. Quantification of lymphocytic choriomeningitis virus with an immunological focus assay in 24- or 96-well plates. J. Virol. Methods 33, 191-198 (1991).

66. Marillier, R. G., Uyttenhove, C., Goriely, S., Marbaix, E. \& Van Snick, J. IL27 p28 is essential for parent-to-F1 acute graft-versus-host disease. Eur. J. Immunol. 44, 2064-2073 (2014)

67. Satija, R., Farrell, J. A., Gennert, D., Schier, A. F. \& Regev, A. Spatial reconstruction of single-cell gene expression data. Nat. Biotechnol. 33 495-502 (2015).

68. Kowalczyk, M. S. et al. Single-cell RNA-seq reveals changes in cell cycle and differentiation programs upon aging of hematopoietic stem cells. Genome Res 25, 1860-1872 (2015).

69. Picelli, S. et al. Full-length RNA-seq from single cells using Smart-seq2. Nat. Protoc. 9, 171-181 (2014).

70. Trapnell, C. et al. Differential gene and transcript expression analysis of RNAseq experiments with TopHat and Cufflinks. Nat. Protoc. 7, 562-578 (2012).

\section{Acknowledgements}

We are grateful to Dr. Casey Weaver from University of Alabama at Birmingham for providing us 10BiT mice. This work is supported by NIH grants AI125741 (W.C.), 5T32HL007209 (R.Z), DK108557 (D.M.S), R37AR40072 (J.C.), R21AR068662 (J.C.), P30AR053495 (J.C.), HL126166 (W.R.D.), and by American Cancer Society (ACS) Research Scholar Grant (W.C). R.Z. is also supported by the Cancer Research Institute Irvington Fellowship. G.X. is supported by The Elizabeth Elser Doolittle Postdoctoral Fellowship. D.M.S. is a member of the Medical Scientist Training Program at MCW, which is partially supported by a training grant from NIGMS T32-GM080202.

\section{Author contributions}

G.X., R.Z., D.M.S. and Y.C., and W.C. performed the experiments and analyzed the data D.M.S. analyzed RNA-seq datasets. J.S.W., W.R.D., V.T., and J.C. provided helpful 
insight and contributed with key reagents. J.C. provided IL-21-tRFP mice. W.C. and R.Z. supervised the study and wrote the paper.

\section{Additional information}

Supplementary Information accompanies this paper at https://doi.org/10.1038/s41467018-07492-4.

Competing interests: The authors declare no competing interests.

Reprints and permission information is available online at http://npg.nature.com/ reprintsandpermissions/

Publisher's note: Springer Nature remains neutral with regard to jurisdictional claims in published maps and institutional affiliations. (c) (i) Open Access This article is licensed under a Creative Commons Attribution 4.0 International License, which permits use, sharing, adaptation, distribution and reproduction in any medium or format, as long as you give appropriate credit to the original author(s) and the source, provide a link to the Creative Commons license, and indicate if changes were made. The images or other third party material in this article are included in the article's Creative Commons license, unless indicated otherwise in a credit line to the material. If material is not included in the article's Creative Commons license and your intended use is not permitted by statutory regulation or exceeds the permitted use, you will need to obtain permission directly from the copyright holder. To view a copy of this license, visit http://creativecommons.org/ licenses/by/4.0/.

(C) The Author(s) 2018 